\title{
Diffusionsbildgebung: Technik und klinische Anwendung
}

Guido Matthias Kukuk, Susanne Greschus, Jan Goldstein, Claus Christian Pieper

\author{
Während die Diffusionsbildgebung früher überwiegend zur Diagnostik der zere- \\ bralen Ischämie eingesetzt wurde, hat sie mittlerweile ein breites Indikations- \\ spektrum in der MRT-Diagnostik. Insbesondere die onkologische Bildgebung, \\ zunehmend aber auch entzündliche Erkrankungen gehören mittlerweile zu den \\ Haupteinsatzgebieten. Die vorliegende Arbeit befasst sich mit den Grundlagen \\ der Diffusionsbildgebung und ihren wichtigsten Indikationen.
}

\section{Grundlagen und Technik}

\section{Diffusion}

\section{Was ist Diffusion?}

Die Diffusion ist die gegenseitige Durchdringung von Gasen, Flüssigkeiten oder Festkörpern durch die Relativbewegung der jeweils enthaltenen Teilchen. Die bekannteste Wirkung der Diffusion ist die Vermischung verschiedener Gase oder Flüssigkeiten unter Abbau eines Konzentrationsgradienten in einem geschlossenen System. Auch im thermodynamischen Gleichgewicht, in dem es keinen Netto-Fluss gibt, führen die Teilchen aufgrund ihrer thermischen Energie ungerichtete Zufallsbewegungen aus, die auch Brown'sche Molekularbewegung genannt werden. Die Teilchenbewegung in einer Substanz ohne Konzentrationsgefälle bezeichnet man auch als Selbstdiffusion.

\section{Isotrope Diffusion}

Für uneingeschränkte Beweglichkeit der Moleküle (isotrope Diffusion) besteht nach dem Fick-Gesetz ein linearer Zusammenhang zwischen dem Mittelwert des Quadrats der Teilchenverschiebung $x(t)$ nach gegebener Zeit $t$ und dem Diffusionskoeffizienten D ( $D$ hängt von Faktoren wie z. B. Druck, Temperatur und den beteiligten Substanzen ab):

$\left[x(t)^{2}\right]=2 d D t$

wobei d die Dimension des Systems ist.

Die Verteilung der Moleküle folgt dabei einer GaußNormalverteilung.

\section{Diffusion im menschlichen Körper}

Der menschliche Körper besteht zum Großteil aus Wasser. Die Wassermoleküle im menschlichen Körper diffundieren jedoch nur teilweise frei. Zum einen werden sie durch Körperbestandteile wie Zellmembranen oder Makromoleküle an der ungestörten Diffusion gehindert, zum anderen werden Moleküle in Blut- oder Lymphgefäßen mit unterschiedlich hohen Geschwindigkeiten transportiert. Wenn der Diffusion Barrieren entgegenstehen, wird die Bewegung der Teilchen zu einem gewissen Grad eingeschränkt. Es ist dann nicht mehr von einer Gauß-Normalverteilung auszugehen. Der Grad der Abweichung von der Normalverteilung wird durch die Diffusionskurtosis quantifiziert.

\section{Anisotrope Diffusion}

Im Extremfall von gerichtet organisierten Geweben (z. B. Nervenbahnen) ist Diffusion praktisch nur entlang dieser Strukturen möglich und damit ebenfalls gerichtet (anisotrope Diffusion). In diesem Fall hängt D von der Raumrichtung ab, in der gemessen wird. Die Richtungsabhängigkeit der Diffusion wird mathematisch mit dem Diffusionstensormodell beschrieben. Durch Messung der Diffusion in mindestens 6 Raumrichtungen ist es möglich, die Richtung und Größe der kleinsten, mittleren und größten Diffusion zu ermitteln und darüber hinaus die mittlere Diffusivität (MD) sowie das Ausmaß der Anisotropie des Gewebes (fraktionelle Anisotropie, FA) zu bestimmen. Diese Parameter finden Anwendung in der Diffusions-Tensor-Bildgebung (DTI).

Für biologische Gewebe liegt die mittlere Diffusivität bei $1 \mu \mathrm{m}^{2} / \mathrm{ms}$. Für eine Diffusionszeit von z.B. $50 \mathrm{~ms}$ liegt die mittlere zurückgelegte Strecke eines Moleküls damit in der Größenordnung von zellulären Strukturen. Es ist daher möglich, über die Messung entsprechender Diffusionseffekte Informationen über die Architektur des untersuchten Gewebes zu erhalten. 


\section{Diffusion in der MRT}

Wird das MRT-Signal durch Anwendung entsprechender Sequenzen besonders anfällig für Diffusionseffekte gemacht, spricht man von diffusionsgewichteter Bildgebung, kurz DWI („diffusion weighted imaging“).

\section{b-Werte und ADC}

Bei der Standardsequenztechnik nach Stejskal und Tanner werden starke Magnetfeldgradienten vor und nach einem Inversionsimpuls geschaltet. Der 1.Gradient dient dabei zur Dephasierung der Spins. Blieben alle Spins am gleichen Ort, würden sie durch den 2.Gradienten wieder vollständig rephasiert. Kommt es aber in der Zwischenzeit durch Diffusion zu einem Ortswechsel der Spins, ist die Rephasierung unvollständig und man kann einen Signalabfall messen. Das gemessene Signal S(b) hängt dabei vom Diffusionskoeffizienten $\mathrm{D}$ des Gewebes und den technischen Parametern, mit denen eine Diffusionswichtung erzeugt wird, ab. Diese Parameter (gyromagnetisches Verhältnis, Gradientenstärke, Gradientendauer, Diffusionszeit) werden im Allgemeinen als b-Wert zusammengefasst, sodass unter Annahme eines mono-exponentiellen Zusammenhangs für das MR-Signal gilt:

$\mathrm{S}(\mathrm{b})=\mathrm{S}_{0} \mathrm{e}^{-\mathrm{bD}}$

Um D bestimmen zu können, müssen also mindestens 2 Messungen mit unterschiedlichen b-Werten durchgeführt werden. Da die Diffusion der Wassermoleküle in vivo eingeschränkt ist und neben den b-Werten von weiteren Faktoren abhängt, bezeichnet man den gemessenen Koeffizienten als erkennbaren Diffusionskoeffizienten, kurz ADC (,apparent diffusion coefficient").

Für klinische Anwendungen hat sich die Verwendung von b-Werten zwischen 0 und $2000 \mathrm{~s} / \mathrm{mm}^{2}$ etabliert, meist werden b-Werte bis $1000 \mathrm{~s} / \mathrm{mm}^{2}$ verwendet, um ein ausreichendes Signal-zu-Rausch-Verhältnis (SNR, „signal-to-noise ratio“) zu gewährleisten. In Geweben mit kurzen T2-Relaxationszeiten kann das SNR dennoch problematisch niedrig werden. Insbesondere in Geweben mit langen T2-Relaxationszeiten kann es dagegen zum sog. T2-Durchschein-Effekt kommen (T2-shinethrough-Effekt).

Die Wahl der optimalen b-Werte einer Untersuchung hängt ab von:

- dem zu untersuchenden Organ

- der klinischen/wissenschaftlichen Fragestellung

- den technischen Möglichkeiten des MRT-Systems

\section{Einflussfaktoren}

Neben der Diffusion beeinflussen auch andere Effekte das Signal in der DWI. In stark vaskularisierten Organen gilt dies vor allem für den Blutfluss. Er ist schneller als die Diffusionsbewegung. In den kleinen Blutgefäßen gilt er dem gängigen Modell zufolge als pseudozufällig und wird daher auch als Pseudodiffusion bezeichnet. In Erweiterung des mono-exponentiellen Modells wurde daher ein bi-exponentielles Modell zur Beschreibung dieser Effekte vorgeschlagen, das auch als IVIM-Modell („intravoxel incoherent motion“) bezeichnet wird. Das Signal S(b) hängt dabei nicht nur vom echten Diffusionskoeffizienten D, sondern auch vom Pseudodiffusionskoeffizienten $D^{*}$ und der Perfusionsfraktion $f$ ab:

$S(b)=S_{0}\left[f e^{-b D^{*}}+(1-f) e^{-b D}\right]$

Dabei ist D* i.d.R. deutlich größer als D.

\section{Einsatzmöglichkeiten der DWI}

Aufgrund dieser hier nur grob skizzierten komplexen Abhängigkeiten haben sich die Strategien der DWI in den vergangenen Jahren in verschiedene Richtungen entwickelt. Je nach anatomischer Region, untersuchtem Organ bzw. untersuchter Erkrankung, beabsichtigter Aussagekraft und technischen Voraussetzungen unterscheiden sich DWI-Protokolle verschiedener Institutionen oft stark voneinander. Eine Vereinheitlichung der Standards wäre in vielerlei Hinsicht wünschenswert, diese steht für die meisten Indikationen allerdings noch aus.

Möglichkeiten, um die DWI im klinischen Alltag oder zu wissenschaftlichen Zwecken sinnvoll einzusetzen, sind:

- für das schnelle Auffinden von diffusionseingeschränkten Arealen: Akquisition von 2 b-Werten (z. B. 0 und $800 \mathrm{~s} / \mathrm{mm}^{2}$ ) und die Erstellung einer ADC-Karte

- für eine hohe Sensitivität beim Auffinden von Läsionen: DWI mit einem niedrigen b-Wert $>0$ bis $200 \mathrm{~s} / \mathrm{mm}^{2}$ (z. B. $50 \mathrm{~s} / \mathrm{mm}^{2}$ ); Blutgefäße zeigen einen hohen Signalverlust („black-blood images“)

- für die quantitative (IVIM-)Analyse unter Berücksichtigung von Perfusions- und Diffusionseinflüssen: Akquisition sowohl niedriger (einschließlich 0) als auch hoher b-Werte

- für die Analyse von Diffusionsrichtungen, die anatomischen Strukturen folgen: Akquisition von mindestens 2 b-Werten mit Gradienten in mindestens 6 unterschiedlichen Raumrichtungen, um den Diffusions-Tensor berechnen zu können

- für die Untersuchung, welchen Einfluss die Diffusionsrestriktion durch Zellmembranen (intrazellulärer und extrazellulärer Raum) hat: zusätzliche Aufnahmen bei sehr hohen b-Werten zur Berechnung der Diffusionskurtosis („diffusion kurtosis imaging“, DKI) 


\section{Sequenzen}

Prinzipiell können verschiedene Sequenztypen angepasst werden, um Diffusionseffekte zu messen. In der klinischen Anwendung kommen derzeit vor allem 2 zum Einsatz:

\section{Spin-Echo-EPI-DWI}

Die Single-Shot-Spin-Echo-EPI-Sequenz (EPI = „echo planar imaging“) wird am häufigsten zur Diffusionsbildgebung eingesetzt ( $\boldsymbol{A} \mathbf{A b b} \mathbf{b}$ ). Dank technischer Verbesserungen (z.B. stärkere Gradienten, parallele Bildgebung, partielle Fourier-Rekonstruktion) kann diese Sequenz auch für die abdominelle Bildgebung eingesetzt werden. Die Sequenzen sind jedoch anfällig für Suszeptibilität, was zu Bildverzerrungen sowie zu Signalverlusten und/oder -überhöhungen führen kann. Problematisch sind Regionen mit hohen Suszeptibilitätsunterschieden, also vor allem luftgefüllte Räume und Knochen-Weichteil-Grenzen. Technische Maßnahmen wie die parallele Bildgebung können diese Probleme in gewissem Maße reduzieren.

\section{Turbo-Spin-Echo- oder Fast-Spin-Echo-DWI}

Eine Alternative sind TSE-DWI-Sequenzen (TSE = TurboSpin-Echo), die weniger anfällig für Suszeptibilitätsartefakte sind. Sie sind in ihrem Auflösungsvermögen und SNR nicht optimal, jedoch werden lokale Inhomogenitäten besser ausgeglichen. Die TSE-DWI-Sequenz ist häufig eine gute Wahl im Bereich luftgefüllter Räume oder an Grenzen von Knochen und Weichgewebe wie an der Schädelbasis oder am Felsenbein ( $\bullet$ Abb.2).

\section{Alternative Sequenzen}

Weitere Sequenzen, die seltener eingesetzt werden, sind z. B. kombinierte Gradienten- und Spin-Echo-Sequenzen (GRASE) oder Steady-State-Free-PrecessionSequenzen (SSFP).

\section{Bewegungskontrolle}

Physiologische Bewegungsprozesse wie Atmung oder Herzschlag können die diffusionsbedingten Molekularbewegungen überlagern. Hierdurch kann es zu zusätzlichen Signalabfällen kommen. Häufig angewendete Techniken, um Bewegungsartefakte zu reduzieren, sind eine Atem- und/oder Herzfrequenztriggerung oder -navigation. So kann auch in schwierigen anatomischen Regionen eine diagnostische Bildqualität mit gutem SNR erzielt werden-allerdings erhöhen diese Techniken die Akquisitionszeit in Abhängigkeit von der Atem- und/oder Herzfrequenz.

Alternativ kann man auch während eines Atemstillstands messen, wodurch sich Bewegungsartefakte bei kooperationsfähigen Patienten verringern lassen. Erzielbares SNR, Auflösung und die Anzahl der messbaren b-Werte sind bei dieser Variante jedoch begrenzt.

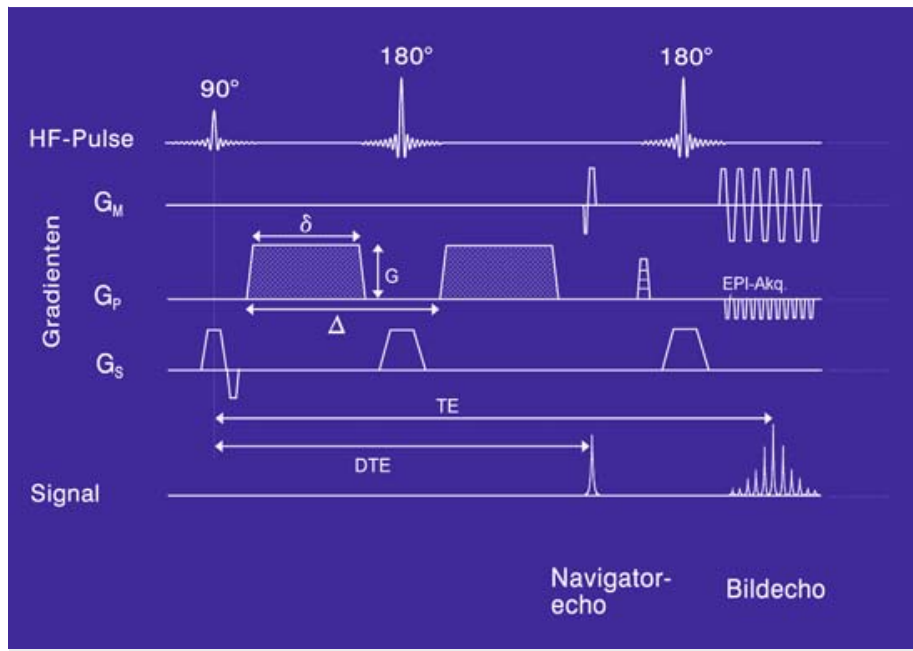

- Abb. 1 Zeitlicher Ablauf einer diffusionsgewichteten Spin-Echo-Bildgebungssequenz (Stejskal-Tanner-Sequenz). Die Diffusionswichtung wird mithilfe eines starken Magnetfeldgradienten (G) erzeugt, der im gleichen Abstand vor und hinter dem Invertierungspuls einer $90^{\circ}-180^{\circ}$ HF-Pulskombination angelegt wird. Die Protonenspins werden dadurch zunächst de- und anschließend rephasiert, was aber aufgrund des Ortswechsels der Wassermoleküle nicht vollständig möglich ist. So kommt es zu einer Signalabschwächung, die umso stärker ist, je mehr sich die Wassermoleküle bewegen („apparent diffusion coefficient“, ADC) und je höher die Diffusionswichtung ist. Die Diffusionswichtung wird vom sog. b-Wert bestimmt, der von der Stärke G der Diffusionsgradienten, der Dauer $\delta$ und dem Abstand $\Delta$ der Magnetfeldgradienten sowie vom gyromagnetischen Verhältnis y abhängt. Das Navigatorecho enthält Phasenvariationen aufgrund von Bewegungen. Das Kernspinsignal wird i. d. R. mittels echoplanarer Bildgebung ausgelesen (EPI-Akq.). $\mathrm{GM}=$ Gradient Ausleserichtung, $\mathrm{GP}=\mathrm{Gradient}$ Phasenkodierrichtung, $\mathrm{GS}=$ Gradient Schichtselektion, DTE = Echozeit Navigatorecho, $\mathrm{TE}=$ Echozeit Bildecho.
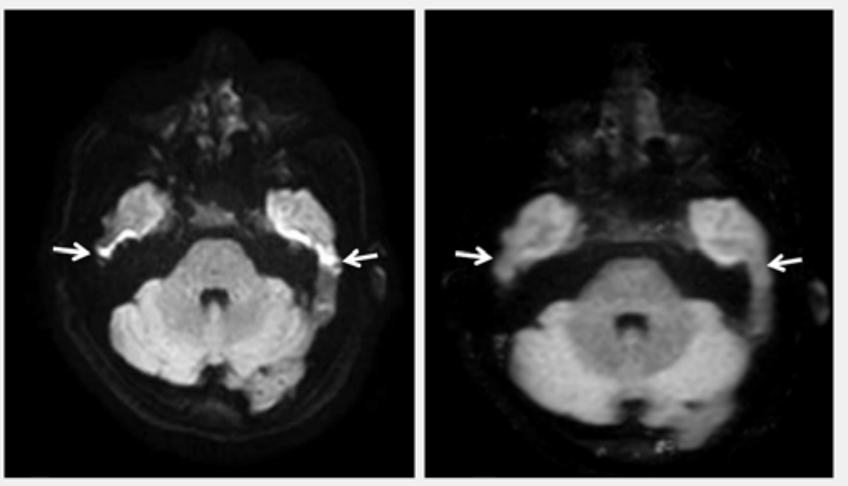

- Abb. 2 Das Spin-Echo-EPI-DWI-Bild (links) mit einem b-Wert von $1000 \mathrm{~s} / \mathrm{mm}^{2}$ zeigt typische Artefakte an der Luft-Knochen-Grenze im Bereich des Felsenbeins (Pfeile). Diese Artefakte sind in der TSE-DWISequenz (b-Wert ebenfalls $1000 \mathrm{~s} / \mathrm{mm}^{2}$ ) deutlich verringert (rechtes Bild). 


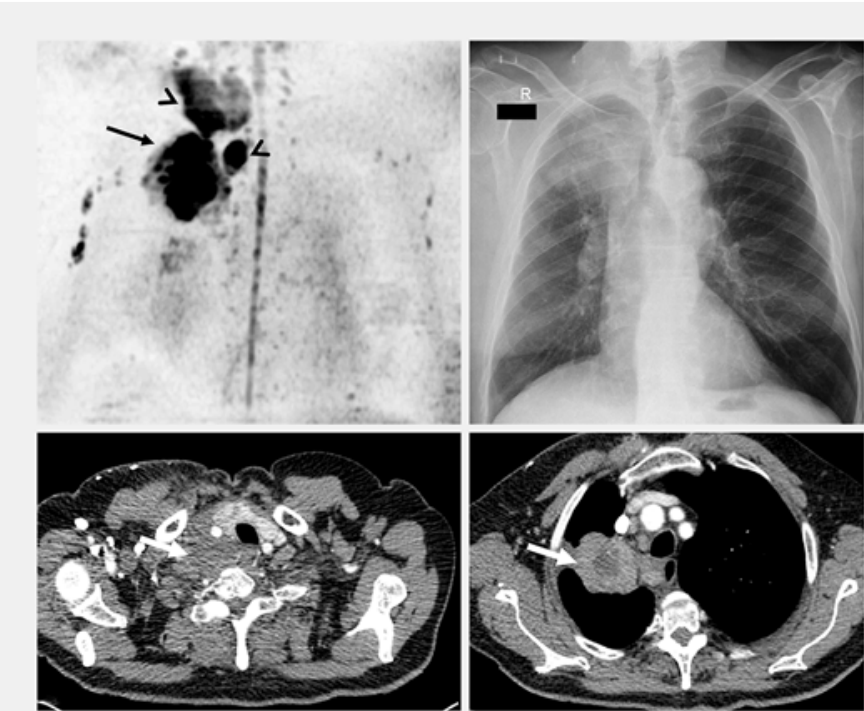

- Abb. 3 Bronchialkarzinom im rechten Oberlappen. In der DWIBS (oberes linkes Bild) Nachweis des Karzinoms (Pfeil) und von mediastinalen sowie zervikalen Lymphknotenmetastasen (Pfeilspitzen). Im Vergleich dazu der Röntgen-Thorax des Patienten (oberes rechtes Bild). In der CT (unteres linkes Bild) Nachweis des Primärtumors (Pfeil) und bei Kontrastmittelverstärkung der Lymphknotenmetastasen (Pfeil) (unteres rechtes Bild).

\section{Fettsuppression}

Da Fett starke Artefakte im DWI-Bild verursachen kann, ist die Fettsuppression im Rahmen der DWI wichtig. Entscheidend sind Zuverlässigkeit, kurze Aufnahmezeiten und ein hohes SNR. Die meist verwendeten Techniken sind spektrale Fettunterdrückungstechniken wie die Spectral Presaturation by Inversion Recovery (SPIR) oder Spectral Attenuated Inversion Recovery (SPAIR). Ein Vorteil der SPAIR-Technik ist der homogenere Fettsuppressionsimpuls vor allem bei B1-Feldinhomogenitäten, wie sie bei höheren Feldstärken auftreten. Der SPAIR-Impuls geht mit einer Verlängerung der Scanzeit einher. Beide Techniken erfordern ein auf die Untersuchungsregion angepasstes Shimming. Moderne Shimming-Techniken können die Bildqualität deutlich verbessern. Eine Alternative ist die Fettsuppression mit der STIR-Technik. Hierbei ist meist eine längere Messzeit erforderlich (größere Anzahl von „signal averages“), da die STIR-Technik im Vergleich zu den spektralen Techniken sonst nur mit einem geringeren SNR umsetzbar ist.

\section{DWIBS}

Die STIR-Technik spielt auch im Rahmen der Diffusionsbildgebung mit Körper-Hintergrund-Unterdrückung („diffusion-weighted whole-body imaging with background body signal suppression“, DWIBS) für die diffusionsgewichtete Untersuchung großer Körperregionen eine Rolle. Üblicherweise handelt es sich bei der DWIBS um eine STIR-EPI-DWI-Sequenz, mit der eine dünn- schichtige Bildakquisition mit folgender 3D-Rekonstruktion möglich ist. Die Daten können auch in freier Atmung erfasst werden. Bei der DWIBS wird i.d. R. keine ADC-Karte erstellt. Die Analyse findet meist visuellqualitativ an invertierten Bildern statt ( $\triangleright$ Abb. $\mathbf{3})$. Diffusionseingeschränkte Läsionen erscheinen dann schwarz vor hellem Hintergrund, der Bildeindruck ist vergleichbar zum PET. Häufig werden Post-ProcessingTechniken wie die Maximum-Intensitätsprojektion (MIP) oder das Volumen-Rendering angewendet.

Wegen ihrer hohen Sensitivität für Strukturen mit hoher Zelldichte umfassen die klinischen Anwendungsmöglichkeiten der DWIBS insbesondere:

- Ganzkörper-Staging bei Lymphomen

- Verlaufskontrolle von Lymphomen unter Therapie

- Entdeckung metastasensuspekter Läsionen

- Tumornachweis bei unklarem Primarius

Ein ungelöstes Problem der DWIBS ist ihre eher niedrige Spezifität. Daher ist eine Korrelation mit weiteren morphologischen bzw. kontrastmittelverstärkten Sequenzen wichtig.

\section{DWI im ZNS}

\section{Allgemeines}

Historisch gesehen verdankt die Diffusionsbildgebung ihren Durchbruch der zuverlässigen Darstellung ischämischer Schlaganfälle. Für viele Jahre blieb ihr klinischer Einsatz auf die zerebrale Bildgebung beschränkt. Durch zunehmende Anwendung fanden sich im Verlauf weitere Hirnerkrankungen, die ebenfalls mit Veränderungen in der Diffusionsbildgebung einhergingen und diagnostisch von großem Wert sind. Die Diffusionsbildgebung des Gehirns stützt sich i.d.R. auf einen niedrigen und einen hohen b-Wert (z.B. $0 \mathrm{~s} / \mathrm{mm}^{2}$ und $1000 \mathrm{~s} / \mathrm{mm}^{2}$ ).

\section{Ischämischer Schlaganfall}

Dass der ischämische zerebrale Insult mit einer Einschränkung der Diffusion einhergeht, ist hinlänglich bekannt und vielfach nachgewiesen ( $\triangleright$ Abb.4). Als pathophysiologisches Korrelat der Diffusionsstörung wird in einer vereinfachten Vorstellung das irreversible zytotoxische Ödem und damit der Kern des Infarkts angesehen [1]. Dieses Modell trifft auch weitgehend auf den subakuten, d. h. den Infarkt zu, der mindestens 6 Stunden und bis zu mehrere Tage alt ist. Beim akuten Infarkt ist es jedoch nur eingeschränkt gültig, denn Diffusionsstörungen können durch eine effektive Rekanalisierung zerebraler Arterien teilweise reversibel sein, wie sich in Thrombektomiestudien gezeigt hat [2]. Der ADC-Wert erreicht sein Minimum typischerweise nach 1-4 Tagen, danach steigt er an und es tritt eine Pseudonormalisierung nach 7-15 Tagen ein. Das DWI-Bild 
kann aufgrund eines T2-shine-through-Effekts in diesem Stadium hyperintens bleiben ( $\triangleright$ Tab.1).

\section{Merke}

Diffusionsstörungen im akuten zerebralen Infarkt können reversibel sein. DWI-Läsion und Infarktkern sind nicht zwingend identisch.

\section{Status epilepticus}

Eine Differenzialdiagnose bei Nachweis von Diffusionsstörungen in der MRT ist der Status epilepticus (Fallbeispiel, - Abb.5). Bei diesem Krankheitsbild können Auffälligkeiten in der Diffusionswichtung ohne und mit Absenkung des ADC ähnlich wie bei einem Infarkt auftreten. Wesentliche differenzialdiagnostische Kriterien liefern zum einen die klinischen Informationen und zum anderen das meist spezifische Verteilungsmuster der Diffusionsstörungen. Beim Status epilepticus liegen die Läsionen oft einseitig auf den Kortex beschränkt, das Marklager aussparend, supratentoriell und lassen sich keinem Gefäßterritorium zuordnen. Typischerweise sind Hippokampus und posteriorer Thalamus beteiligt. Falls die Diagnose aus der klinischen Information und dem Verteilungsmuster nicht eindeutig hervorgeht, kann eine Messung der Perfusion die nötige Zusatzinformation erbringen. Hirnareale mit einer gesteigerten Exzitation haben einen vermehrten Sauerstoffbedarf, der mit einer im Seitenvergleich vermehrten Perfusion einhergeht. Die Diffusionsrestriktion liegt also in demselben Areal wie die gesteigerte Perfusion, während bei der Ischämie die diffusionsgestörte Region weniger gut durchblutet ist. Die Diffusionsstörungen beim Status epilepticus sind meist reversibel, können jedoch auch zu einer dauerhaften Schädigung (z. B. Hippokampussklerose) führen.

\section{Merke}

Diffusionsrestriktionen beim Status epilepticus liegen vor allem kortikal und im limbischen System.

\section{Entzündliche Läsionen}

Eine weitere sinnvolle Anwendung der DWI ist die Darstellung entzündlicher Läsionen. Der zerebrale Abszess ist - wie Abszesse an anderen Stellen auch - typischerweise durch eine verminderte Diffusion gekennzeichnet, die aus dem hohen Proteingehalt der Flüssigkeit resultiert ( $\mathbf{A}$ bb.6). In der MRT zeigt sich demnach eine zystische Läsion, deren Inhalt diffusionsgestört ist. Vorsicht ist jedoch nach einer Blutung und bei einem postoperativen Situs geboten: Das in einer Blutungs- oder Seromhöhle liegende Blut kann vor allem in frühen Stadien ebenfalls eine Diffusionsrestriktion aufweisen. Klärung können eine SWI oder T2* Sequenz in Zusammenschau mit den Standardsequenzen
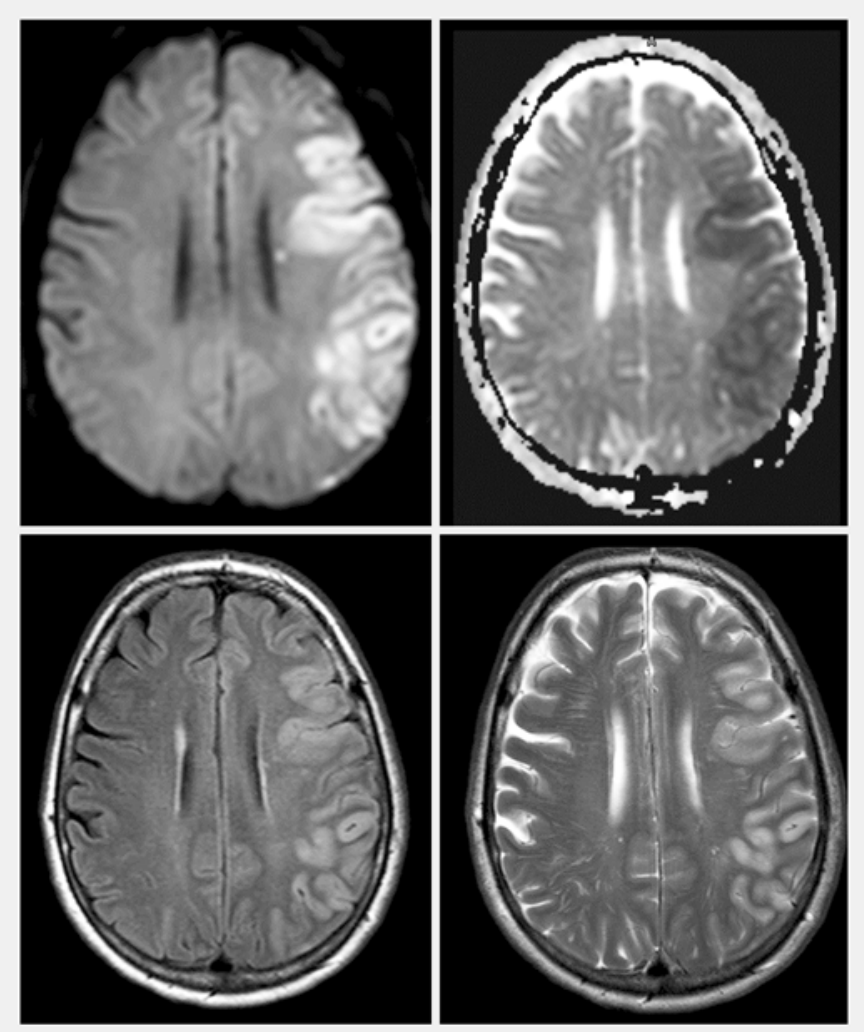

- Abb.4 Linkshemisphärischer Infarkt nach Verschluss der A. cerebri media am Vortag. Das zytotoxische Ödem ist in der Diffusionsbildgebung (oberes linkes Bild, b-Wert $1000 \mathrm{~s} / \mathrm{mm}^{2}$ ) und der ADC-Karte (oberes rechtes Bild) zu erkennen. Auch in der FLAIR- (unteres linkes Bild) und in der T2-Wichtung (unteres rechtes Bild) demarkiert sich der Infarkt in Form eines kortikalen und subkortikalen Ödems im linksseitigen Media-Versorgungsgebiet.

\ Tab. 1 Diffusionsbildgebung des ischämischen Schlaganfalls.

\begin{tabular}{|c|c|c|}
\hline Infarktalter & $\begin{array}{l}\text { Diffusionsbildgebung } \\
\text { (hoher b-Wert) }\end{array}$ & ADC \\
\hline$<6$ Stunden & $\begin{array}{l}\text { hyperintens (selten bei } \\
<1 \text { Stunde alten Infarkten } \\
\text { isointens) }\end{array}$ & vermindert \\
\hline $6-24$ Stunden & hyperintens & vermindert \\
\hline 1 - 7 Tage & hyperintens & $\begin{array}{l}\text { vermindert (ADC-Werte } \\
\text { steigen aber langsam an) }\end{array}$ \\
\hline 1 - 8 Wochen & $\begin{array}{l}\text { hyperintens/isointens } \\
\text { (ab ca. } 10 . \text { Tag dominiert } \\
\text { T2-shine through-Effekt) }\end{array}$ & normal bis erhöht \\
\hline $\begin{array}{l}\text { Monate bis } \\
\text { Jahre }\end{array}$ & $\begin{array}{l}\text { isointens (evtl. hyperintens } \\
\text { durch T2-shine-through- } \\
\text { Effekt) }\end{array}$ & meist erhöht \\
\hline
\end{tabular}




\section{FALLBEISPIEL}

\section{Verdacht auf epileptischen Anfall}

Der Notarzt bringt eine 56-jährige Frau unter der Verdachtsdiagnose eines epileptischen Anfalls in die Notaufnahme. Den Anfall konnte der Notarzt mit i.v. Thiopental durchbrechen, die Patientin ist intubiert, beatmet und wird tracheal abgesaugt. Anamnestisch sind eine arterielle Hypertonie und ein Alkoholabusus bekannt, außerdem ein Suizidversuch vor einigen Jahren. Es besteht der Verdacht auf einen Medikamentenabusus. Die regelmäßige Medikation ist unbekannt.
Bei Aufnahme sind die Augen der Patientin geöffnet, es besteht eine leichte Pupillendifferenz mit rechts erweiterter Pupille. Nystagmus nach links. Noch leichte Zuckungen am linken Arm und linken Bein. Auf Schmerzreiz allseits gezielte Abwehr. In der CT lassen sich weder eine Blutung noch eine akute Ischämie nachweisen, in der CT-Perfusion findet sich kein Perfusionsdefizit. Die Aufnahmen der MRT sind in $>$ Abb. $\mathbf{5}$ gezeigt. Es wird die Diagnose eines Status epilepticus gestellt.
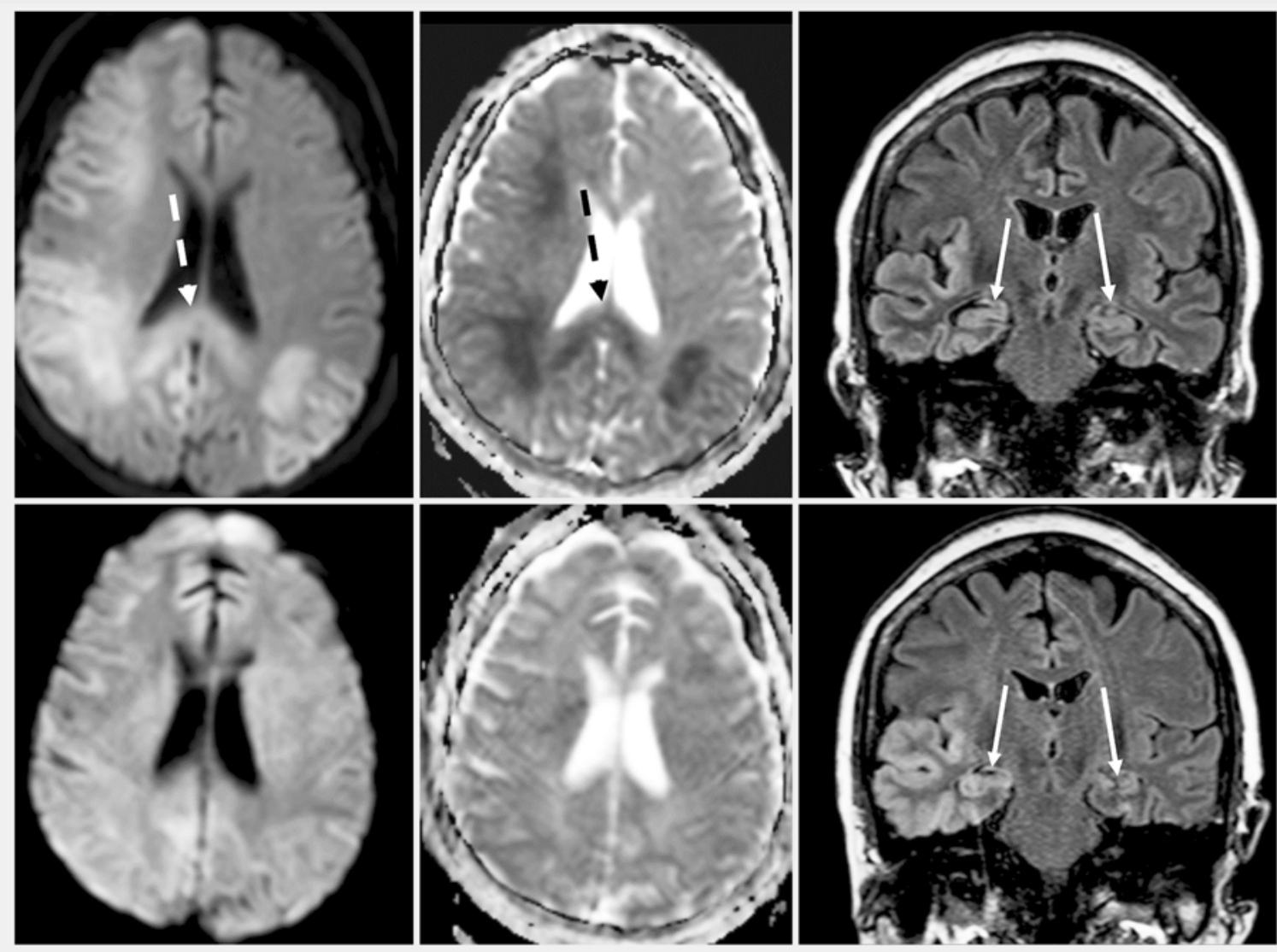

- Abb.5 Verlauf bei Status epilepticus. Ein Tag nach dem Erstereignis (obere Bilder) finden sich kortikale und subkortikale Signalanhebungen in der DWI ( $b=1000 \mathrm{~s} / \mathrm{mm}^{2}$, oberes linkes Bild), die ausweislich der ADC-Karte (oberes mittleres Bild) nur z.T. einem zytotoxischen Ödem entsprechen. Charakteristisch für die Diffusionsstörungen im Status epilepticus sind die Läsionen im Splenium des Balkens (gestrichelte Pfeile). Ebenfalls typisch ist die Beteiligung des limbischen Systems mit Ödem der Hippocampi (oberes rechtes Bild, FLAIR-Wichtung, Pfeile), der Inselrinde und des Temporallappens. Nach einer Woche (untere Bilder) sind die Diffusionsstörungen (unteres linkes und mittleres Bild) zwar rückläufig, das beidseitige kortikale Ödem in der FLAIR-Wichtung besteht jedoch immer noch (unteres rechtes Bild, Pfeile).

(T1- und T2-Wichtung) herbeiführen, mittels derer sich das Blut als solches nachweisen lässt.

Eine viral bedingte Infektion, die mit typischen verteilten Diffusionsstörungen einhergeht, ist die Herpes-Enzephalitis. Die Diffusionsstörungen gehen im frühen
Stadium dem in der FLAIR- oder T2-Wichtung sichtbaren Ödem voraus. Somit ist die DWI in diesem Fall die sensitivste und wichtigste Sequenz in der Abklärung. Aufgrund der starken Affinität der Herpesviren zu den Neuronen und der Glia des limbischen Systems finden sich die diffusionsgestörten Areale an typischer Stelle: 
temporal (insbesondere im Hippokampus), Inselrinde, subfrontaler Kortex, Gyrus cinguli, im späteren Verlauf auch im übrigen Kortex. Häufig ist ein beidseitiger, jedoch asymmetrischer Befall des supratentoriellen Gehirns. Die Läsionen liegen kortikal und subkortikal mit relativer Aussparung der weißen Substanz und sind damit den Veränderungen beim Status epilepticus sehr ähnlich. Zur Differenzierung ist je nach klinischer Situation die Klärung mittels Liquordiagnostik erforderlich. Die Diffusionsstörungen bei der Herpes-Enzephalitis münden meist in eine Nekrose und damit in persistierende Läsionen -im Gegensatz zu den meist reversiblen Läsionen beim Status epilepticus.

\section{Differenzialdiagnostik von Raumforderungen}

In der Differenzialdiagnostik primärer intrakranieller Neoplasien und Hirnmetastasen hat die Diffusionsbildgebung noch keinen etablierten Stellenwert. Der ADC-Wert ist näherungsweise invers proportional zur Tumorzelldichte, allerdings wird er auch durch andere Faktoren wie Nekrosen und Perfusion beeinflusst. Bei den primären ZNS-Neoplasien gilt eine substanzielle Überlappung der ADC-Werte von 0,82-2,73× $10^{-3} \mathrm{~mm}^{2} / \mathrm{s}$, in einigen Studien konnte man jedoch im Gewebe bzw. Ödem um Metastasen höhere ADC-Werte messen als bei primären ZNS-Tumoren [3]. ZNS-Lymphome haben dagegen eher niedrige ADC-Werte, bei immunsupprimierten Patienten kann man mit dem ADC-Wert manchmal ein Lymphom von einer Toxoplasmose unterscheiden. Allerdings gibt es auch hier eine große Überlappung der ADC-Werte - ein ADC-Wert unter $0,8 \times 10^{-3} \mathrm{~mm}^{2} / \mathrm{s}$ spricht jedoch eher für ein Lymphom [4]. Zur Abgrenzung von Epidermoid- gegen Arachnoidalzysten ist die DWI dagegen sehr gut geeignet: Epidermoidzysten zeigen im Gegensatz zu den Arachnoidalzysten eine deutliche Diffusionsrestriktion und erscheinen somit hyperintens im DWI-Bild.

\section{DWI der Kopf-Hals-Region}

\section{Allgemeines}

Der Fokus liegt im Kopf-Hals-Bereich vor allem auf der Beurteilung tumoröser Prozesse. Zusätzlich zu den morphologischen Sequenzen ist es mit der DWI möglich, Tumoren zu unterscheiden, den Lymphknotenstatus im Rahmen des Stagings zu erheben und das Ansprechen von Tumoren auf eine Therapie zu beurteilen.

\section{Merke}

In der Kopf-Hals-Region gibt es verschiedene, in der DWI hypertense Gewebearten. Dazu gehört vor allem das lymphatische Gewebe des Waldeyer-Rachenrings, die Nerven des Plexus brachialis und die meist reichlich vorhandenen Lymphknoten.
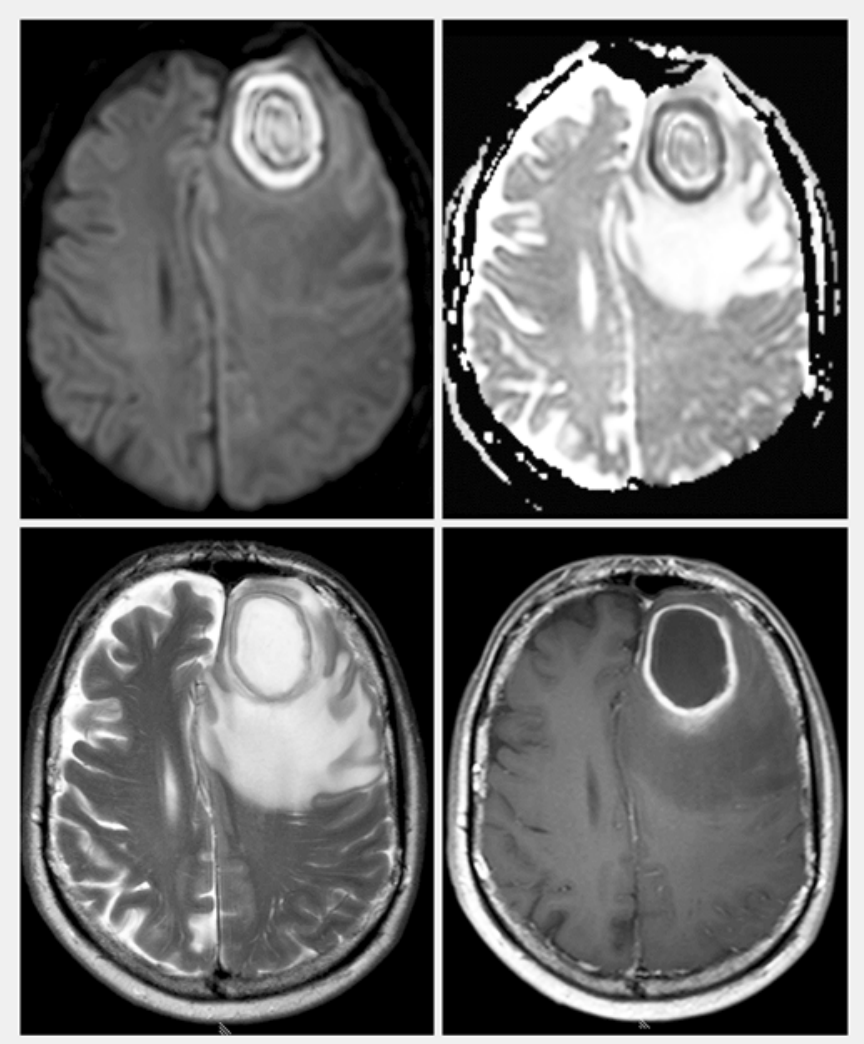

Abb.6 53-jähriger Patient mit linksfrontalem Hirnabszess. Eine Diffusionsstörung ist im DWI-Bild (oberes linkes Bild, $b=1000 \mathrm{~s} / \mathrm{mm}^{2}$ ) und in der ADC-Karte (oberes rechtes Bild) der zentralen Anteile und der Abszesskapsel zu sehen. Die T2- (unteres linkes Bild) und die T1-Wichtung (unteres rechtes Bild) weisen die zentrale Flüssigkeit und eine stark kontrastmittelanreichernde Abszesskapsel (unteres rechtes Bild) nach. Typischerweise ist die Kapsel in der T2-Wichtung (unteres linkes Bild) breit und hypointens.

\section{MR-Neurografie}

Den hohen Organisationsgrad von Nervenfasern nutzt man bei der Diffusionstensorbildgebung (DTI) zur Darstellung der Faserbündel. Hiermit kann man den Verlauf der Nervenfasern im Sinne einer „Neurografie“ in der MRT darstellen und z.B. die Integrität der Fasern nach einer Nervenverletzung prüfen.

\section{Tumoren}

Bei einer Tumorerkrankung eignet sich die Diffusionsbildgebung zum einen für die Suche nach Lymphknoten. Diese können dann unter Berücksichtigung der übrigen Sequenzen anhand ihrer Größe, der Struktur und dem möglichen Vorhandensein von Nekrosen weiter differenziert werden. Der Befall von Lymphknoten durch Metastasen von Tumoren im Kopf-Hals-Bereich (vor allem Plattenepithelkarzinomen) führt häufig zu einer deutlichen Absenkung des ADC-Wertes. So gelten Werte des ADC unter $0,94 \times 10^{-3} \mathrm{~mm}^{2} / \mathrm{s}$ als starker Prädiktor eines Lymphknotenbefalls (84\% Sensitivität, 

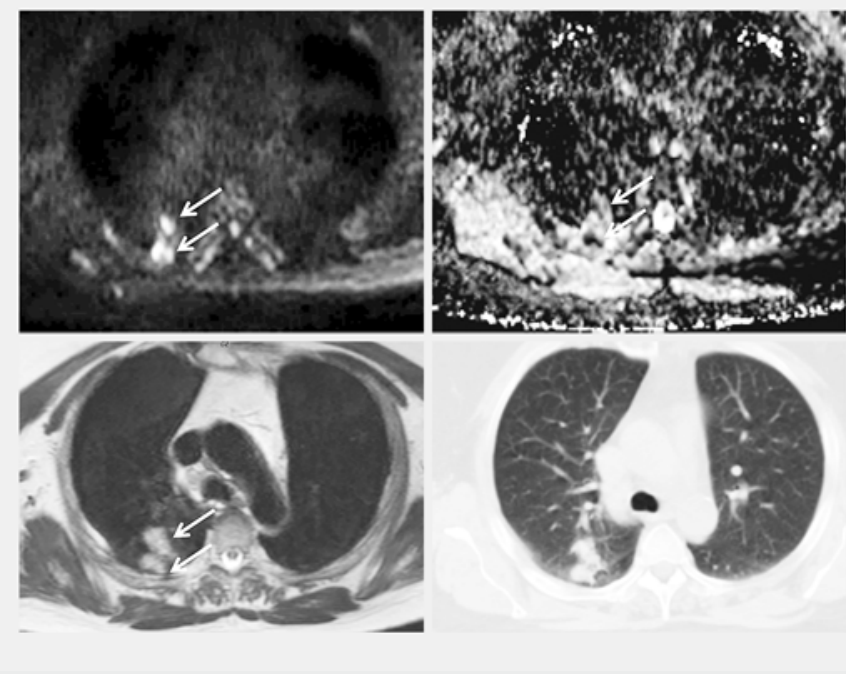

- Abb. 7 Hochdifferenziertes Adenokarzinom des rechten Lungenoberlappens (Differenzierungsgrad G1) bei einer 55-jährigen Patientin mit langjährigem Nikotinabusus. Das diffusionsgewichtete Bild (oberes linkes Bild) mit einem b-Wert von $1000 \mathrm{~s} / \mathrm{mm}^{2}$, aufgenommen an einem 3-Tesla-MRT, zeigt einen hyperintensen Tumor mit 2 soliden Arealen (Pfeile). Die korrespondierende ADC-Karte (oberes rechtes Bild) berechnet aus den b-Werten 0, 50, 250 und $1000 \mathrm{~s} / \mathrm{mm}^{2}$. Der Tumor-ADC beträgt etwa $0,5 \times 10^{-3} \mathrm{~mm}^{2} / \mathrm{s}$. Auf der T2w Aufnahme (unteres linkes Bild) stellt sich der Tumor ebenfalls dar (Pfeile), das untere rechte Bild zeigt die entsprechende CT der Patientin im Lungenfenster.

$94 \%$ Spezifität) [5]. Die ADC-Werte hängen jedoch von verschiedenen weiteren Faktoren ab (u. a. Typ der verwendeten Sequenz, b-Werte) und sollten daher nur ein Anhaltspunkt sein. Dies gilt insgesamt für die Tumordiagnostik anhand von ADC-Werten, da die DWI aktuell keinen einheitlichen Standards unterworfen ist. Ferner muss berücksichtigt werden, dass nicht alle Tumorarten eine Diffusionseinschränkung aufweisen.

\section{Entzündliche Veränderungen}

Wie im Gehirn liefert die Diffusionsbildgebung wichtige Informationen bei entzündlichen Prozessen. Die Diffusion ist in der Flüssigkeit einer Abszesshöhle deutlich eingeschränkt, bei phlegmonösen Entzündungen jedoch nicht gestört. Ebenso wenig sind andere Flüssigkeiten diffusionsgestört, wie z. B. die Flüssigkeit in einer Halszyste oder Tumornekrosen.

\section{Thorax}

\section{Allgemeines}

Aufgrund von Herz- und Atembewegungen, Gefäßpulsationen, geringer Protonendichte und großen Unterschieden in der magnetischen Suszeptibilität ist die diffusionsgewichtete Bildgebung der Thoraxorgane technisch anspruchsvoll und artefaktanfällig. Unter Einsatz atemgetriggerter Sequenzen und vorzugsweise eines modernen 1,5-Tesla-Scanners ist jedoch auch die thorakale DWI durchführbar. Höhere b-Werte (z.B. b=
$1000 \mathrm{~s} / \mathrm{mm}^{2}$ ) sind aufgrund des Einflusses der Perfusion in pulmonalen Läsionen sinnvoll.

\section{Merke}

Probleme der Thoraxregion sind Bewegungen und große Unterschiede in der Suszeptibilität der enthaltenen Organe. Die thorakale DWI ist mit den derzeit verfügbaren Geräten durchführbar und erlaubt auch den Einsatz hoher b-Werte.

\section{Pulmonale Raumforderungen}

Zu den häufigsten Fragestellungen in der thorakalen Bildgebung gehören die Suche nach pulmonalen Rundherden und die Beurteilung der Dignität von inzidentell diagnostizierten Läsionen. Die CT als Goldstandard besitzt zwar eine ausgezeichnete Sensitivität, zeigt aber Schwächen bei der Unterscheidung zwischen malignen und benignen Läsionen. Die PET-CT weist eine relativ hohe Rate von falsch positiven Befunden insbesondere bei inflammatorischen Veränderungen auf.

Die kontrastmittelverstärkte MRT der Lunge zeigte in einigen Studien eine gute Spezifität auch im Vergleich zur Multidetektor-CT. Welchen Nutzen die DWI in der Diagnostik pulmonaler Läsionen hat, kann noch nicht abschließend beurteilt werden. Bei Läsionen über $6 \mathrm{~mm}$ liegt ihre Sensitivität bei immerhin $86 \%$, bei Läsionen bis zu $5 \mathrm{~mm}$ dagegen unter $50 \%$ [6]. Unklar ist noch, ob die DWI zur Unterscheidung zwischen benignen und malignen pulmonalen Herden eingesetzt werden kann. Verschiedene Metaanalysen kamen diesbezüglich zu gegensätzlichen Ergebnissen. Auch die Frage, ob anhand von ADC-Werten Hinweise auf die Tumorart oder den Tumordifferenzierungsgrad gewonnen werden können, ist noch Gegenstand der Forschung. Gut differenzierte Adenokarzinome zeigten in einer Studie zwar höhere ADC-Werte als gering differenzierte Adenokarzinome, diese ersten Ergebnisse sind auf den Einzelfall allerdings noch nicht anwendbar ( $\triangleright$ Abb. 7). Ein Vorteil der DWI im Vergleich zur T2w Sequenz besteht darin, dass die DWI ähnlich wie die PET-CT zwischen Tumorgewebe und poststenotischer Atelektase unterscheiden kann [7].

\section{Merke}

Bislang ist die Datenlage bezüglich der Fähigkeit der DWI, zwischen benignen und malignen Rundherden zu differenzieren, uneinheitlich. In einigen Fällen kann die DWI nützlich zu sein, um Tumorgewebe von poststenotischen Atelektasen abzugrenzen.

\section{Pleura}

In einer Studie erleichterte die DWI die prognostisch wichtige Unterscheidung zwischen dem epithelialen und dem sarkomatoiden Subtyp des Pleuramesothe- 
lioms, wobei der sarkomatoide Typ eher einen niedrigeren ADC hatte [8]. Exsudative Pleuraergüsse zeigten im Vergleich zu transsudativen signifikant geringere ADC-Werte und ließen sich mit einer Sensitivität von ca. $90 \%$ und einer Spezifität von ca. $85 \%$ diagnostizieren [9]. Diese Ergebnisse sind allerdings noch nicht in Folgestudien verifiziert worden.

\section{Mediastinum}

Der ADC-Wert kann Hinweise auf die Dignität von Raumforderungen des Mediastinums geben. Während maligne Läsionen wie Lymphome, invasive Thymome oder das Bronchialkarzinom eher einen niedrigen ADCWert aufweisen, lassen sich in der retrosternalen Struma, im nicht invasiven Thymom oder in der Thymus-Rebound-Hyperplasie eher höhere ADC-Werte messen. In Bezug auf das Lymphom-Staging liegen für die DWI ähnlich gute Ergebnisse wie für die CT oder die PET-CT vor. Für die Darstellung des thorakalen Ösophaguskarzinoms eignet sich die DWI bislang nicht.

\section{Herz}

Die Herzbildgebung gehört aufgrund der Bewegungen zu den technisch anspruchsvolleren Anwendungen der DWI. Unter Verwendung bestimmter Sequenztypen, einer Atemtriggerung und einer Synchronisierung auf die Diastole gibt es zu diesem Thema erste Studien. Bislang wurde eher mit niedrigen b-Werten (bis $400 \mathrm{~s} / \mathrm{mm}^{2}$ ) gearbeitet. Mögliche zukünftige Indikationen sind die Differenzierung von nekrotischem und vitalem Myokard im Rahmen der Ischämie-Abklärung, die Myokarditisdiagnostik, die Differenzialdiagnose von Herztumoren sowie die Quantifizierung myokardialer Fibrosen.

\section{Merke}

Mögliche Anwendungsgebiete der thorakalen DWI sind vor allem die nicht invasive Diagnostik pulmonaler, mediastinaler und kardialer Erkrankungen.

\section{Leber}

\section{Allgemeines}

Diffusionsgewichtete Sequenzen gehören mittlerweile zu den Standardsequenzen in der Leberbildgebung. Sie spielen eine wichtige Rolle in der Differenzialdiagnostik von fokalen und diffusen Lebererkrankungen und für die Therapiekontrolle bei malignen Erkrankungen. Die Bilder werden i.d.R. atemgetriggert akquiriert. Die Wahl der b-Werte hängt von den technischen Möglichkeiten und der beabsichtigten Auswertung ab und umfasst meist Werte zwischen 0 und $1000 \mathrm{~s} / \mathrm{mm}^{2}$. Mindestens ein niedriger $\left(0-100 \mathrm{~s} / \mathrm{mm}^{2}\right)$ und ein höherer $\left(500-1000 \mathrm{~s} / \mathrm{mm}^{2}\right)$ b-Wert sollten jedoch immer aufgenommen werden.
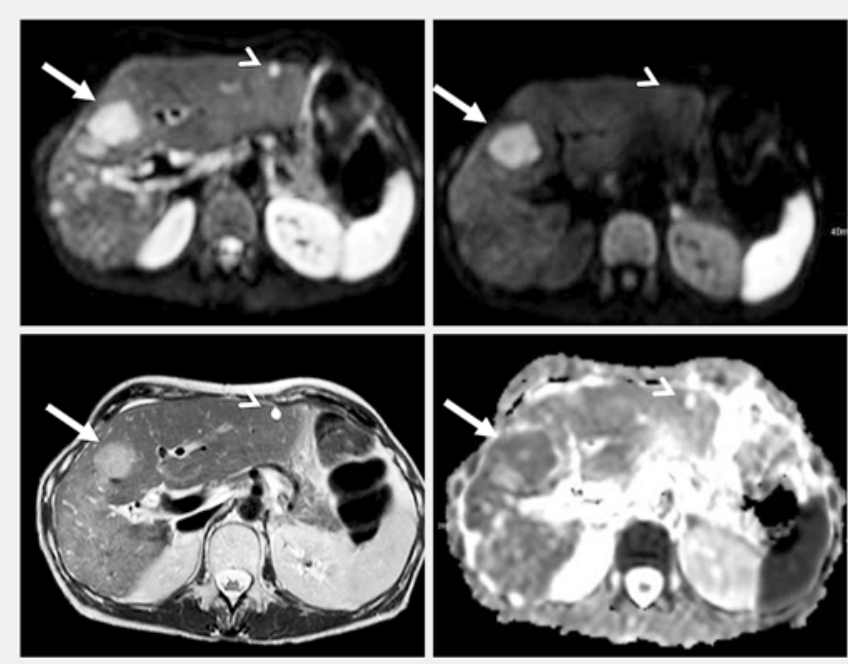

Abb.8 62-jährige Patientin mit Gewichtsverlust. Das diffusionsgewichtete Bild mit einem b-Wert von $50 \mathrm{~s} / \mathrm{mm}^{2}$ (oberes linkes Bild) zeigt 2 hyperintense Leberläsionen (Pfeil und Pfeilspitze). Bei einem b-Wert von $800 \mathrm{~s} / \mathrm{mm}^{2}$ (oberes rechtes Bild) verschwindet die kleinere Läsion (Pfeilspitze). Auf der T2w Aufnahme (unteres linkes Bild) werden beide Läsionen dargestellt. Ausweislich der ADC-Karte (unteres rechtes Bild) beträgt der ADC-Wert der Metastase (Pfeil) etwa $1,0 \times 10^{-3} \mathrm{~mm}^{2} / \mathrm{s}$, während der ADC-Wert der Zyste (Pfeilspitze) bei etwa $2,9 \times 10^{-3} \mathrm{~mm}^{2} / \mathrm{s}$ liegt.

\section{Merke \\ In der Leberbildgebung sind DWI-Bilder mit niedrigen b-Werten $\left(0<b<100 \mathrm{~s} / \mathrm{mm}^{2}\right)$ gut geeignet, um Leber- läsionen nachzuweisen. Zur Charakterisierung von Lebererkrankungen sind auch höhere b-Werte und eine ADC-Auswertung erforderlich.}

\section{Nachweis von Leberläsionen}

Der Nachweis von Leberläsionen ist die am besten etablierte und am weitesten verbreitete Indikation. Leberläsionen sind als hyperintens zum umliegenden Lebergewebe und bereits auf diffusionsgewichteten Aufnahmen mit niedrigem b-Wert gut abzugrenzen. Auf diffusionsgewichteten Aufnahmen mit höheren b-Werten zeigen zystische Läsionen bereits einen Signalverlust, während solide Raumforderungen sich aufgrund ihrer eingeschränkten Diffusion weiterhin gut vom umgebenden Parenchym abheben ( $\mathbf{A b} \mathbf{b} \mathbf{8} \mathbf{8})$. Hinsichtlich des Nachweises von Metastasen ist die DWI anderen Sequenzen gleichwertig oder überlegen $[10,11]$. Die Sensitivität der DWI beim Nachweis von hepatozellulären Karzinomen variiert hingegen stark zwischen den Studien. Die höchste Sensitivität bietet vermutlich eine Kombination aus DWI und einer kontrastverstärkten Sequenz. 
Tab. 2 DWI/ADC-Verhalten verschiedener Läsionen.

\begin{tabular}{|l|l|l|l|}
\hline Läsion & $\mathbf{b}=\mathbf{0} \mathbf{s} / \mathbf{m m}^{\mathbf{2}}$ & $\mathbf{b}>\mathbf{5 0 0} \mathbf{s} / \mathbf{m m}^{\mathbf{2}}$ & $\mathbf{A D C}$ \\
\hline $\begin{array}{l}\text { benigne Läsion } \\
\text { (z.B. Zyste) }\end{array}$ & hyperintens & hypointens & hoch \\
\hline $\begin{array}{l}\text { T2-shine-through-Effekt } \\
\text { (z.B. Zyste, Hämangiom) }\end{array}$ & hyperintens & hyperintens & hoch \\
\hline $\begin{array}{l}\text { maligne Läsion } \\
\text { (z.B. Metastase) }\end{array}$ & hyperintens & hyperintens & niedrig \\
\hline
\end{tabular}

Merke

Die höchste Sensitivität beim Nachweis von Leberläsionen bietet eine Kombination aus DWI und kontrastmittelverstärkter MRT.

\section{Charakterisierung von Leberläsionen}

Zur Charakterisierung von Leberläsionen ist die Analyse des hohen b-Wert-Bildes und der ADC-Karte erforderlich. Kommt es bei hohen b-Werten zu einem Signalabfall der Läsion, handelt es sich meist um eine benigne Läsion ( $\$$ Tab.2). Hämangiome stellen sich häufig hyperintens auf dem DWI-Bild mit hohem b-Wert dar, haben jedoch i.d.R. einen hohen ADC-Wert (T2-shinethrough-Effekt). Bleibt die Läsion hyperintens und hat einen niedrigen ADC-Wert, muss an Malignität gedacht werden. Zwischen fokal-nodulären Hyperplasien, Adenomen und malignen Läsionen gibt es einen größeren Überlappungsbereich, sodass eine ADC-Bestimmung alleine nicht ausreicht, um die Läsion zu charakterisieren. Durch Analysen von Aufnahmen mit vielen b-Werten (IVIM) kann sich die Charakterisierung der Läsion verbessern.

Merke

Bei der Beurteilung der Dignität einer Leberläsion ist neben der Betrachtung der DWI-Bilder immer auch eine Analyse der ADC-Parameterkarte erforderlich.

\section{Response-Evaluation von Lebertumoren}

Änderungen der ADC-Werte nach systemischer oder lokoregionärer Therapie können einer Tumorgrößenänderung vorausgehen. In ersten Studien war eine erfolgreiche Therapie bei vielen Tumoren mit einem frühen Anstieg der ADC-Werte verbunden. Dieser Anstieg reflektiert vermutlich einen Zerfall des Tumorgewebes [12]. Die Höhe der prätherapeutischen ADC-Werte scheint außerdem bereits vor Einleitung einer Therapie Hinweise auf ein mögliches Therapieansprechen liefern zu können. Da allerdings in einigen Studien höhere Werte mit einer besseren Prognose assoziiert waren, in anderen jedoch niedrigere Werte, sind vermutlich weitere Faktoren wie die Art des Tumors und die Art der Therapie von Bedeutung.

\section{Diffuse Lebererkrankungen}

In den vergangenen Jahren wurde der Stellenwert der Diffusionsbildgebung in der Diagnostik von diffusen Lebererkrankungen wie der Leberfibrose und -zirrhose evaluiert. Im fibrozirrhotischen Leberparenchym sind die ADC-Werte niedriger als im gesunden Lebergewebe, allerdings ist die genaue Beziehung zwischen Fibrosegrad und ADC-Wert noch unklar [13]. In einer Studie stand - bei einem Cut-off-Wert des ADC von $1,21 \times 10^{-3} \mathrm{~mm}^{2} / \mathrm{s}$ - eine Area-under-the-curve (AUC) von 0,92 für den Nachweis einer schweren Fibrose [14].

\section{Gallenwege}

Welchen Stellenwert die DWI bei Erkrankungen der Gallenwege hat, ist bisher nur wenig untersucht. Es lassen sich aber erste Trends zum Einsatz der DWI aus der vorhandenen Literatur ableiten. Die Akquisition von Aufnahmen mit hohen b-Werten kann dabei helfen, die Ursache von Gallengangsstrikturen einzuordnen und insbesondere zwischen benignen und malignen Strikturen zu unterscheiden. Die Nachweisrate von extrahepatischen, insbesondere ampullären cholangiozellulären Karzinomen kann ebenfalls durch die zusätzliche Akquisition einer DWI-Sequenz verbessert werden [15].

\section{Pankreas}

\section{Allgemeines}

Zur Diffusionsbildgebung des Pankreas existierende Arbeiten befassen sich vor allem mit dem Nachweis und der Charakterisierung von Adenokarzinomen, neuroendokrinen Tumoren, zystischen Läsionen und der Abgrenzung neoplastischer von entzündlichen Prozessen. Die Diffusionsparameter des gesunden Pankreasgewebes hängen von auch von Alter, Geschlecht und der Organregion ab. Die ADC-Werte nehmen mit dem Alter ab und sind im Pankreaskopf höher als in der Cauda pancreatis. Besondere Bedeutung kann der DWI bei kleinen malignen Läsionen des Pankreas zukommen.

\section{Adenokarzinom des Pankreas und Pankreatitis}

Die klinisch wichtigsten Erkrankungen der Bauchspeicheldrüse sind das Adenokarzinom und die Pankreatitis. Insbesondere die fokale, raumfordernde Pankreatitis kann in der konventionellen Schnittbilddiagnostik oft nicht sicher vom Pankreaskarzinom unterschieden werden. Die DWI kann die Sensitivität beim Nachweis von Pankreaskarzinomen erhöhen ( $\mathbf{A b b . 9}$ ). Da auch die fokale Pankreatitis eine im Verhältnis zum normalen Pankreasgewebe eingeschränkte Diffusion aufweist, bleibt die Spezifität der DWI in dieser Fragestellung jedoch begrenzt [16]. Zudem weisen gut differenzierte Tumoren nicht notwendigerweise niedrigere ADC-Werte als gesundes Pankreasgewebe auf [17]. 
Merke

Die DWI ergänzt die konventionelle kontrastmittelverstärkte MRT des Pankreas vor allem bei soliden Raumforderungen. Verlässliche Daten zur Sensitivität und Spezifität der DWI liegen allerdings noch nicht vor.

\section{Zystische Pankreasläsionen}

Die bisher verfügbaren Daten zur Differenzierung von zystischen Pankreasläsionen sind widersprüchlich, sodass eine breite Anwendung aktuell nicht empfohlen werden kann [18]. Orientierend kann die DWI manchmal Hinweise auf die Dignität der zystischen Läsion liefern. Eine eingeschränkte Diffusion findet sich eher in zystischen Neoplasien, Nekrosen oder Abszessen.

\section{Neuroendokriner Pankreastumor}

Die Sensitivität beim Nachweis sowohl eines primären neuroendokrinen Tumors als auch der entsprechenden Lebermetastasen kann man erhöhen, wenn man die DWI zum Untersuchungsprotokoll hinzufügt. Schlecht differenzierte Tumoren weisen eher niedrigere ADCWerte auf.

\section{Gastrointestinaltrakt}

\section{Allgemeines}

Die MRT der Hohlorgane des Gastrointestinaltrakts wird erschwert durch Atembewegung, Darmperistaltik und Suszeptibilitätsunterschiede. Bewegungsartefakte lassen sich durch eine Atemtriggerung und eine Prämedikation zur Herabsetzung der Darmperistaltik (z.B. Butylscopolamin) reduzieren. Eine Darmdistension mit Flüssigkeit analog zur MR-Enterografie kann die Qualität der DWI entscheidend verbessern, weil Suszeptibilitätsartefakte durch diese Vorbereitung deutlich verringert werden.

\section{Merke}

Suszeptibilitätsartefakte in der Darm-DWI lassen sich durch eine Darmdistension mit Flüssigkeit ähnlich wie bei der MR-Enterografie verringern.

\section{Anorektalregion}

In der Regel kann für die Anorektalregion eine DWI-Sequenz in freier Atmung verwendet werden, da Atembewegungen in dieser Region meist keine Rolle spielen. Medikamente zur Herabsetzung der Darmperistaltik können einen geringen zusätzlichen Qualitätsgewinn bringen. Wichtiger ist die Vermeidung von ausgeprägten Artefakten durch Luft im Rektum. Eine rektale Applikation von Ultraschallgel oder Wasser kann hier Abhilfe schaffen. Pathologische Prozesse werden meist erst bei hohen b-Werten (mindestens $800-1000 \mathrm{~s} / \mathrm{mm}^{2}$ ) sichtbar.

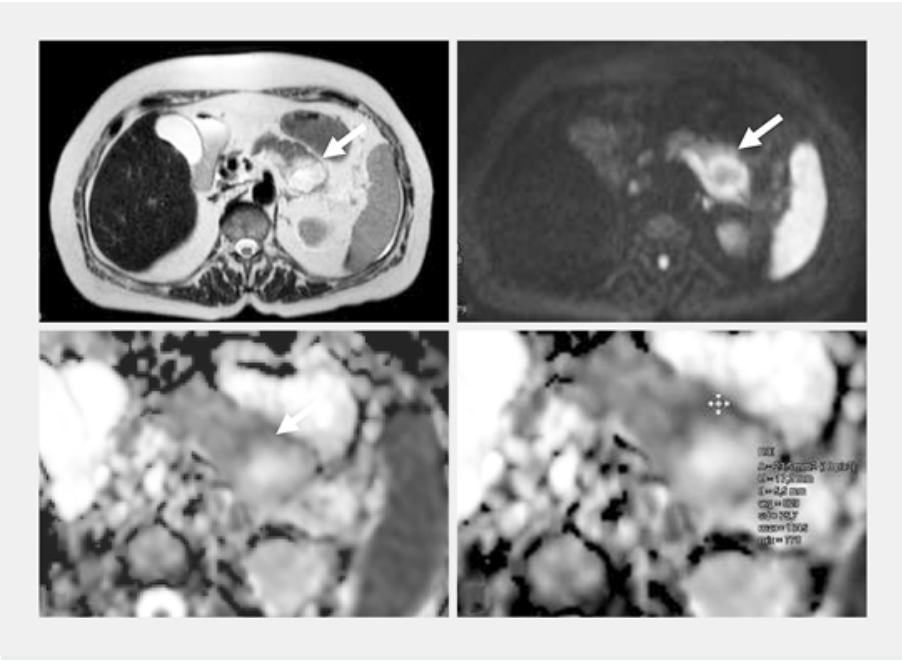

Abb.9 68-jährige Patientin mit Raumforderung des Pankreas. Auf der T2w Aufnahme (oberes linkes Bild) ist eine Raumforderung im Pankreasschwanz (Pfeil) mit Infiltration der V. lienalis zu erkennen. Die Diffusionswichtung (oberes rechtes Bild, b-Wert $800 \mathrm{~s} / \mathrm{mm}^{2}$ ) zeigt einen vor allem im Randbereich diffusionsgestörten, zentral liquiden Tumor (Pfeil). Gemäß ADC-Karte (unteres linkes Bild) liegt der ADC-Wert im Randbereich des Tumors (unteres rechtes Bild) bei etwa $0,9 \times 10^{-3} \mathrm{~mm}^{2} / \mathrm{s}$. Die Histologie erbrachte die Diagnose eines sarkomatoiden Pankreasschwanzkarzinoms.

\section{Merke}

Bei deutlich luftgefüllter Rektumampulle kann die Instillation von Ultraschallgel die Bildqualität entscheidend verbessern.

\section{Entzündliche Darmerkrankungen}

Indikationen zur MR-Enterografie sind vor allem die Diagnostik von chronisch entzündlichen Darmerkrankungen und die Tumorerkennung. Aktiv entzündliche Darmsegmente zeigen ödematöse Veränderungen der verdickten Wand. Zellschwellung und Hyperzellularität führen zu einer reduzierten Diffusion von Wasser in der Darmwand. Erkrankte Darmsegmente zeigen daher eine erhöhte Signalintensität auf diffusionsgewichteten Aufnahmen mit hohen b-Werten ( $\triangleright$ Abb. 10). Die ADC-Wertbestimmung in der Darmwand ist aufgrund der Peristaltik und der Artefakte mit einem größeren Unsicherheitsfaktor behaftet als in den meisten anderen Körperregionen. Etabliert hat sich der Einsatz höherer b-Werte von $800-1000 \mathrm{~s} / \mathrm{mm}^{2}$ [19]. Für die Einschätzung der entzündlichen Aktivität chronisch entzündlicher Darmerkrankungen wie dem Morbus Crohn erreicht die diffusionsgewichtete MRT die Genauigkeit der kontrastmittelverstärkten MRT [19]. Perforationen und Fisteln sollten zusätzlich in konventionellen und kontrastmittelverstärkten Sequenzen beurteilt werden. 

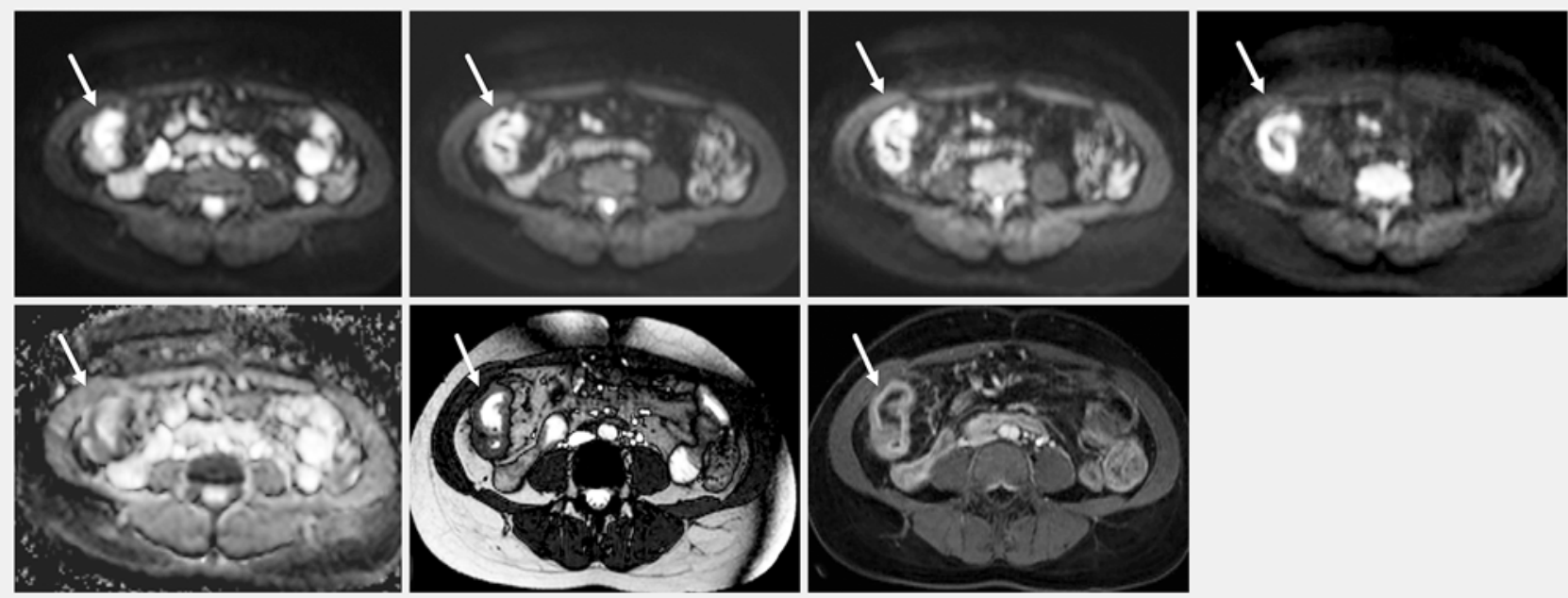

- Abb.10 MRT einer 33-jährigen Patientin mit Verdacht auf Morbus Crohn. Die DWI-Bilder (obere Bilder, b-Werte 0, 50, 250 und 800 s/mm²) zeigen eine mit zunehmenden b-Werten persistierende Signalanhebung der Darmwand im terminalen lleum (Pfeil). Die nicht pathologisch veränderten Darmwände verlieren mit zunehmendem b-Wert an Signal. Die ADC-Karte (unteres linkes Bild) zeigt eine ADC-Absenkung in der Wand des distalen lleums (Pfeil). In der Balanced-FFE (unteres mittleres Bild) und der kontrastverstärkten, fettsupprimierten T1w-Bildgebung (unteres rechtes Bild) sind die Wandverdickung und eine vermehrte Kontrastmittelanreicherung der Darmwand zu erkennen (Pfeil).

Merke

Die diffusionsgewichtete MR-Enterografie ermöglicht die Beurteilung der entzündlichen Aktivität des Morbus Crohn.

Darmwandfibrosen, wie sie im chronischen Stadium auftreten, können ebenfalls zu einer Absenkung des ADC-Wertes führen. Während bislang noch kein statistisch signifikanter Zusammenhang zwischen histologischem Entzündungsgrad und ADC-Wert nachgewiesen werden konnte, gelang dieser Nachweis im Zusammenhand mit dem Fibrosegrad [20].

Aktiv entzündlich veränderte Darmsegmente unterscheiden sich von chronisch entzündlich-fibrotisch veränderten auch durch ein höheres mikrovaskuläres Volumen. Dieses kann im Rahmen einer IVIM-Analyse nachgewiesen werden. In einer Arbeit trug der „schnelle Anteil“ der Diffusion bei niedrigen b-Werten in aktiv entzündlichen Darmabschnitten stärker zur ADC-Absenkung bei als der „langsame Anteil“ bei hohen bWerten [21]. Wenn diese Ergebnisse reproduziert werden können, erscheint eine Unterscheidung von aktiven und inaktiven fibrotischen Veränderungen anhand der Diffusionsbildgebung möglich.

Merke

Zusätzlich zur üblichen MR-Enterografie kann die DWI die diagnostische Sicherheit verbessern. Zudem können möglicherweise aktive Entzündungsherde von chronisch fibrotischen Veränderungen unterschieden werden.

\section{Maligne Erkrankungen}

Im oberen Gastrointestinaltrakt kann die DWI zur Verbesserung der Tumorerkennung eingesetzt werden. Eine Raumforderung oder Wandverdickung in Kombination mit einer eingeschränkten Diffusion sollte an ein malignes Geschehen denken lassen. Für das Rektumkarzinom erhöht die DWI-Sequenz als Ergänzung zur üblichen T2w Bildgebung die Genauigkeit der Tumorerkennung ( $\mathbf{A}$ Ab. 11). Zudem erleichtert sie den Nachweis von Lymphknotenmetastasen.

\section{Urogenitalsystem}

\section{Allgemeines}

Für die Darstellung des Urogenitalsystems und der Beckenorgane spielt die DWI eine zunehmend wichtige, im Fall der Prostata sogar herausragende Rolle. Wie in anderen Körperregionen auch, liegen die ADC-Werte von Malignomen und Entzündungen der Beckenorgane i.d.R. unterhalb der Werte von Vergleichsgeweben im gesunden Organ. Es muss beachtet werden, dass die Wahl der b-Werte die Grundlage der DWI ist. Es gibt Studien, die durch unterschiedliche Wahl der b-Werte zu völlig verschiedenen Ergebnissen gekommen sind.

\section{Merke}

Die Aussagekraft der DWI wird entscheidend durch die Wahl der b-Werte - der Berechnungsgrundlage des ADC-Wertes - bestimmt. Je nach Erkrankung haben Diffusions- oder Perfusionseffekte einen unterschiedlichen Einfluss. 


\section{Uterus und Zervix}

Je nach zugrunde gelegter Studie und Untersuchungstechnik liegen die ADC-Werte des normalen Myometriums zwischen 1,1 und $1,8 \times 10^{-3} \mathrm{~mm}^{2} / \mathrm{s}$. Die Adenomyose des Uterus als nicht neoplastische, relativ häufige Erkrankung mit ektopem Endometrium innerhalb des Myometriums kann fokal oder diffus auftreten und weist eine niedrige bis mittlere Signalintensität im diffusionsgewichteten Bild auf. Die ADC-Werte lagen einer Studie zufolge unter denen des normalen Myometriums bei etwa $0,86 \pm 0,30 \times 10^{-3} \mathrm{~mm}^{2} / \mathrm{s}$ [22]. Leiomyome als die häufigsten benignen Raumforderungen im Uterus können histologisch teils sehr komplex sein. Dadurch erklärt sich ihre Variabilität in der DWI. Die Spannbreite von ADC-Werten, die in Uterusmyomen gemessen worden sind, lag je nach angewandter Technik zwischen etwa $0,6 \times 10^{-3} \mathrm{~mm}^{2} / \mathrm{s}$ und $1,5 \times 10^{-3} \mathrm{~mm}^{2} / \mathrm{s}$. Degenerierte oder therapierte Leiomyome haben eher höhere ADC-Werte ( $\bullet$ Abb. 12). Uterine Leiomyosarkome als mögliche Differenzialdiagnose degenerierter Myome ließen sich in einer Studie überwiegend anhand ihres niedrigeren ADC-Wertes von den Myomen unterscheiden [23]. Allerdings gibt es, wenn man die derzeitige Literatur betrachtet, noch große Überschneidungen insbesondere auch zu nicht degenerierten Uterusmyomen.

Uterusmyome sind ferner ein Beispiel für gut vaskularisierte Raumforderungen, in denen Perfusionseinflüsse eine große Rolle spielen. Der Therapieerfolg der Myombehandlung mittels hochfokussiertem Ultraschall (HIFU) ließ sich in einer Studie am besten an einem perfusionsgewichteten ADC-Wert ablesen [24].

\section{Merke}

Uterusmyome sind ein gutes Beispiel für gut vaskularisierte Raumforderungen, in denen Perfusionsein-

flüsse eine große Rolle spielen.

Obwohl die MRT zur Diagnostik des Endometriumkarzinoms nicht indiziert ist, war in Studien anhand der ADC-Werte eine Differenzierung zwischen malignen und benignen Läsionen möglich. So lag der ADC-Wert benigner Läsionen in einer Studie immer über 1,28x $10^{-3} \mathrm{~mm}^{2} / \mathrm{s}$. In der Beurteilung der extrauterinen Tumorausdehnung kann die DWI Zusatzinformationen zur Unterscheidung von Tumorgewebe und umgebendem Ödem liefern.

Studien zum Zervixkarzinom fanden überwiegend einen statistisch signifikant niedrigeren ADC-Wert in malignen Läsionen im Vergleich zum nicht betroffenen Gewebe. Mit der DWI lässt sich der Tumor so besser vom umliegenden Gewebe abgrenzen. In einigen Studien korrelierte ferner der histologische Tumorgrad mit dem ADC-Wert, außerdem scheint der ADC-Wert
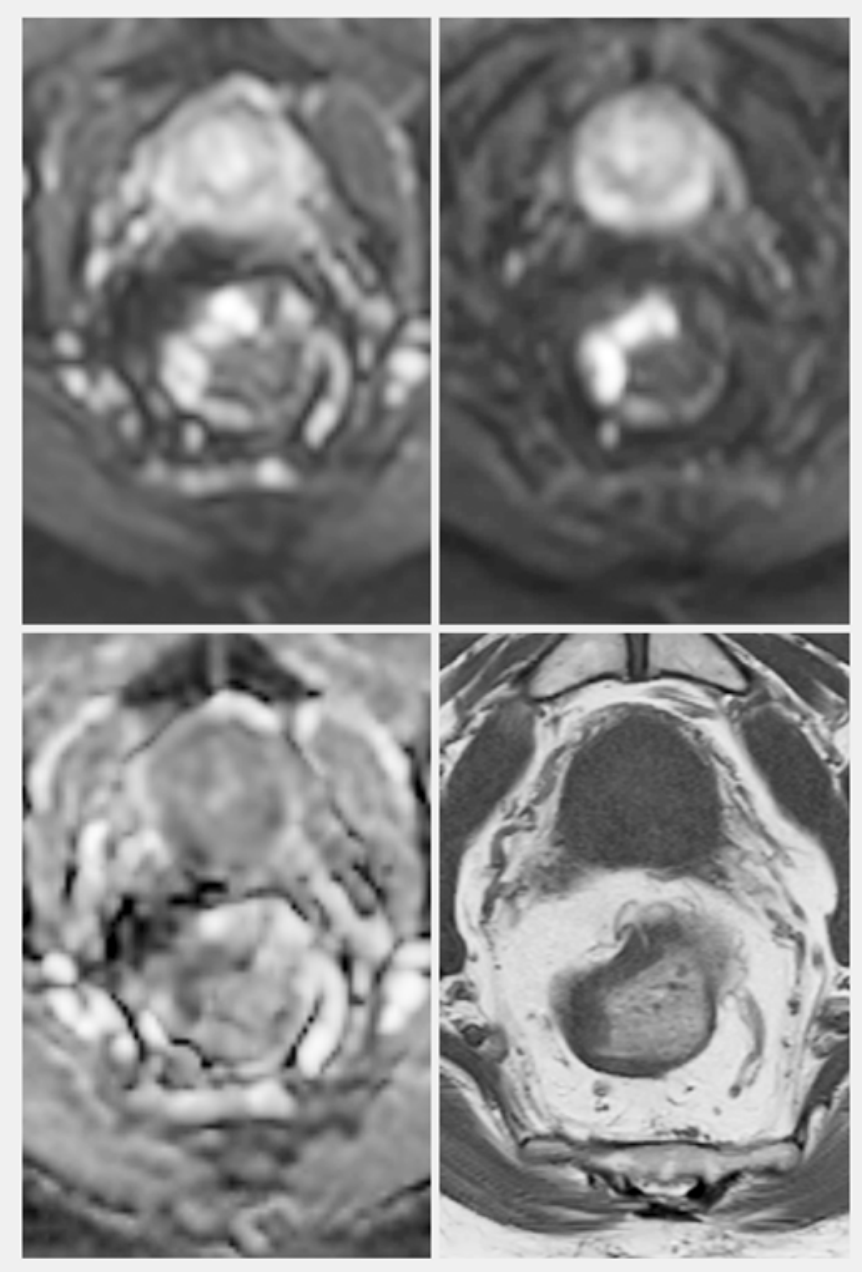

- Abb.11 Semizirkulär wachsendes Rektumkarzinom (cT3, N1) vor Radiochemotherapie. Die DWI-Bilder (obere Bilder, b-Werte 0 und $800 \mathrm{~s} / \mathrm{mm}^{2}$ ) zeigen eine semizirkulär verdickte Wand des Rektums, die sich auch auf dem b800-Bild stark hyperintens darstellt. Außerdem kommt im pararektalen Fettgewebe bei 7 Uhr ein suspekter Lymphknoten zur Darstellung. Ausweislich der ADC-Karte (unteres linkes Bild) ist der ADC-Wert in der Läsion deutlich erniedrigt. Auch in der T2w Aufnahme (unteres rechtes Bild) ist der Tumor darstellbar.

in Plattenepithelkarzinomen niedriger zu sein als in Adenokarzinomen.

Merke

Mittels DWI lässt sich ein Tumor der Cervix uteri besser vom nicht betroffenen oder ödematösen Gewebe abgrenzen.

\section{Ovar und Adnexe}

Die Bildgebung sollte mit mindestens einem niedrigen und einem hohen b-Wert durchgeführt werden (z. B. b 1 $\left.50-100 \mathrm{~s} / \mathrm{mm}^{2}, b_{2} 750-1000 \mathrm{~s} / \mathrm{mm}^{2}\right)$. Für die Bildgebung der Ovarien und der Adnexe liefert die DWI Zusatzinformationen, die manchmal diagnostisch wertvoll sein können. Aufgrund ihrer morphologischen 

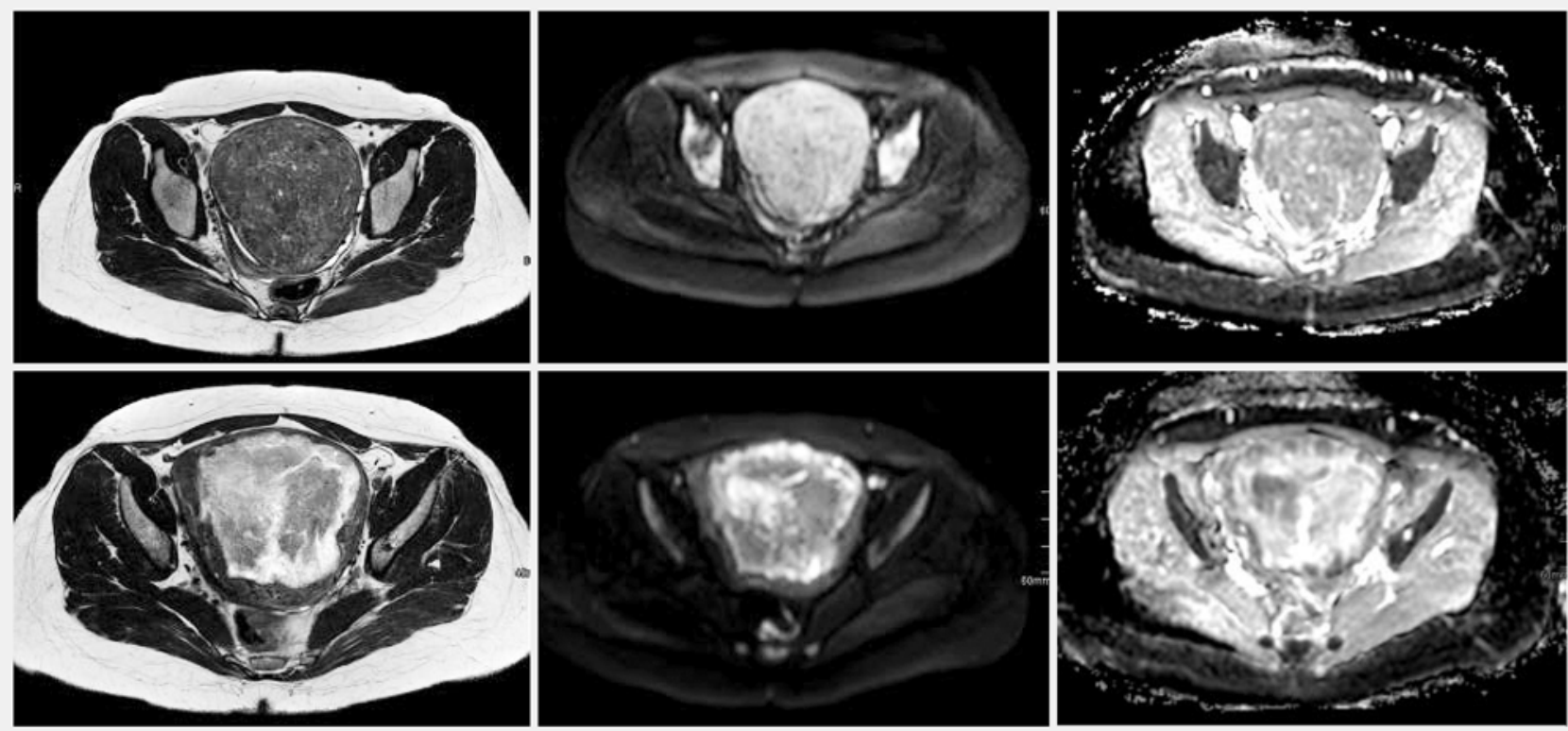

Abb. 12 43-jährige Patientin mit Uterus myomatosus. Das T2w Bild (oberes linkes Bild), das DWI-Bild (oberes mittleres Bild, b=800 s/mm²) und die ADC-Karte (oberes rechtes Bild) zeigen ein großes intramurales Myom der Uterusvorderwand. Der ADC-Wert beträgt $1,4 \times 10^{-3} \mathrm{~mm}{ }^{2} / \mathrm{s}$. Nach der Therapie mit hochfokussiertem Ultraschall (HIFU) sind auf den gleichen Sequenzen (untere Bilder) Zeichen der Kolliquation und ein Anstieg des ADC-Werts auf durchschnittlich $1,8 \times 10^{-3} \mathrm{~mm}^{2} / \mathrm{s}$ zu erkennen.

Heterogenität stellen sich maligne Läsionen in Ovar und Adnexe jedoch völlig unterschiedlich dar, je nachdem, ob zystische Anteile mit niedriger Zellularität, proteinreiche oder eingeblutete Areale oder rein solide Raumforderungen vorliegen. Auf der anderen Seite weisen zahlreiche benigne Raumforderungen wie reife Teratome, Endometriome und funktionelle hämorrhagische Zysten eine Diffusionsrestriktion auf.

Merke

Die DWI ist nicht geeignet, um Läsionen des Ovars und der Adnexe hinsichtlich ihrer Dignität zu beurteilen. Manchmal kann sie aber diagnostisch wertvolle Zusatzinformationen liefern.

Reife zystische Teratome haben meist einen niedrigen ADC-Wert, der u.a. auf die enthaltenen keratinoiden Substanzen zurückzuführen ist, ihre Signalintensität im diffusionsgewichteten Bild (hoher b-Wert) ist daher im Gegensatz zu Zysten deutlich erhöht, der ADC-Wert relativ niedrig ( $\$$ Abb. $\mathbf{1 3}$ ). Auch Endometriosezysten, die Blutabbauprodukte enthalten, zeigen deutlich niedrigere ADC-Werte als andere zystische Läsionen [25].

\section{Niere}

Aufgrund ihrer Wassertransportfunktion, ihrer anatomischen Organisation und ihrer starken Durchblutung sollte die Niere ein für die DWI gut geeignetes Organ sein. Allerdings sind die retroperitoneal gelegenen Nieren starken physiologischen Bewegungen ausgesetzt- die vertikale Atemverschieblichkeit beträgt bis zu $4 \mathrm{~cm}$. In der Regel mitteln sich die atembedingten Akquisitionsfehler jedoch aus, wenn die Patienten gleichmäßig atmen, sodass es möglich ist, die Bildgebung in freier Atmung mit respiratorischer Triggerung durchzuführen. Die gute Durchblutung hat große Auswirkungen auf die DWI und die berechneten ADC-Werte. So führt z.B. eine Pulstriggerung i.d.R. zu zuverlässigeren Messwerten mit geringerer Standardabweichung [26]. Da diese Technik aber mit längeren Akquisitionszeiten verbunden ist, wird sie vielfach nicht eingesetzt.

Die DWI kann neben der diagnostisch führenden Kontrastmitteldynamik hilfreiche Informationen in der Differenzialdiagnostik von Nierentumoren liefern. Bei Nierenzellkarzinomen erleichtert sie häufig die Abgrenzung von Tumorthromben ( $\triangleright$ Abb.14). In einer Metaanalyse hatten Onkozytome einen mittleren ADC-Wert von 2,01 (95\%-Cl 1,84-2,17), während er bei Nierenzellkarzinomen im Mittel bei 1,61 (95\%-Cl $1,45-1,77)$ lag [27]. In der gleichen Studie und in aktuelleren Arbeiten wurde nachgewiesen, dass Angiomyolipome aufgrund ihres hohen Fettgehalts einen niedrigen ADC-Wert aufweisen, der sich teilweise mit dem der Nierenzellkarzinome überschneidet. Angiomyolipome bereiten jedoch in der Differenzialdiagnose aufgrund ihres Fettgehalts i.d. R. kaum Probleme. 

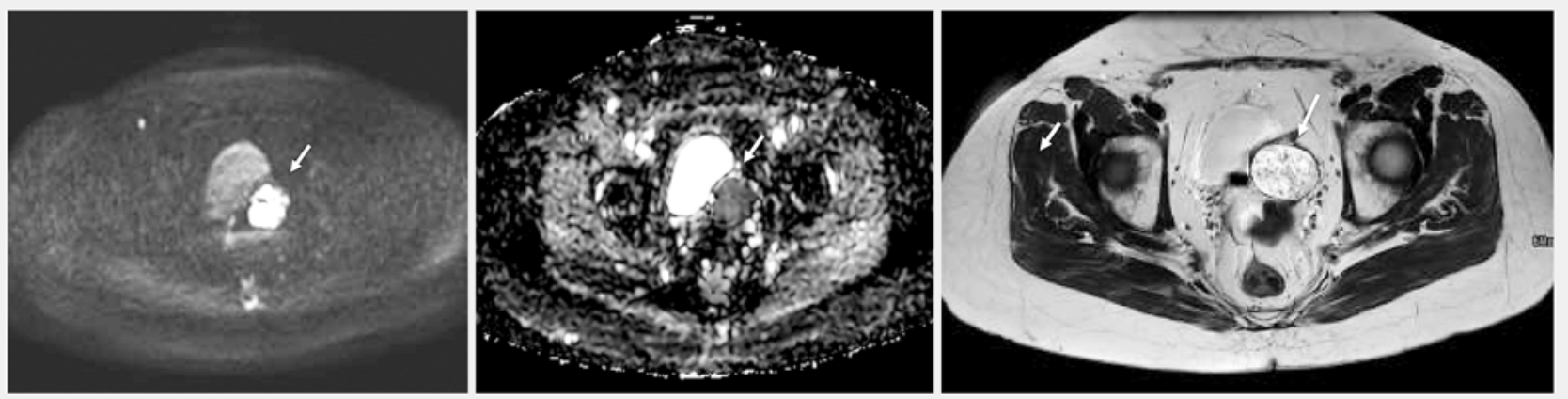

Abb. 13 58-jährige Patientin mit reifem Teratom des linken Ovars. Das DWI-Bild (oberes linkes Bild, b-Wert 1000 s/mm², STIR-Fettunterdrückung) zeigt einen hyperintensen Tumor des linken Ovars (Pfeil). Ausweislich der ADC-Karte (oberes rechtes Bild) hat der Tumor einen niedrigen ADC-Wert (in diesem Fall $0,9 \times 10^{-3} \mathrm{~mm}^{2} / \mathrm{s}$ ), vereinbar mit einem reifen Teratom. Das rechte Bild zeigt die korrespondierende T2w Sequenz. Die Histologie erbrachte die Diagnose eines reifen zystischen Ovarialteratoms (Dermoidzyste) ohne Anhalt für Malignität.

\section{Merke}

Die DWI ergänzt die MRT und CT in der Diagnostik von Nierentumoren. Oft erleichtert sie die Differenzialdiagnostik und kann bei der Abgrenzung von Tumorthromben helfen.

Obwohl Onkozytome einen eher höheren ADC-Wert aufweisen, reichen die in den bisherigen Studien zugrunde gelegten Fallzahlen noch nicht aus, um die Empfehlung zur teilweisen bzw. kompletten Nephrektomie bei soliden Raumforderungen der Niere zu revidieren. Die Überschneidungsbereiche der ADC-Werte zwischen Nierenläsionen sind - je nach Studie - unterschiedlich groß. In einer Metaanalyse aus dem Jahr 2016 wurden die höchsten mittleren ADC-Werte für Zysten gemessen (mittlerer ADC-Wert 2,97), gefolgt von

- normalem Gewebe (mittlerer ADC-Wert 2,48),

- Onkozytomen (mittlerer ADC-Wert 2,04),

- Transitionalzellkarzinomen (mittlerer ADC-Wert 1,94),

- Nierenzellkarzinomen (mittlerer ADC-Wert 1,80) und

- Angiomyolipomen (mittlerer ADC-Wert 1,37) [28].

\section{Prostata}

Im Rahmen der multiparametrischen Bildgebung der Prostata spielt die DWI eine zentrale Rolle. Sie verbessert den Nachweis von Prostatakarzinomen signifikant und ist für die in der Außenzone lokalisierten Tumoren auch die wichtigste Sequenz - noch vor den T2w und den kontrastmittelverstärkten Aufnahmen. In einigen Studien ergaben sich Vorteile für den visuellen Nachweis von Karzinomen unter Verwendung sehr hoher b-Werte von bis zu $2000 \mathrm{~s} / \mathrm{mm}^{2}$. Allerdings bringt die Akquisition solcher Aufnahmen auch Nachteile mit sich - z. B. ein ungünstiges SNR. Die aktuelle PI-RADSv2-Leitlinie empfiehlt die Akquisition eines hohen bWerts von mindestens $800-1000 \mathrm{~s} / \mathrm{mm}^{2}$. Eine mögli-
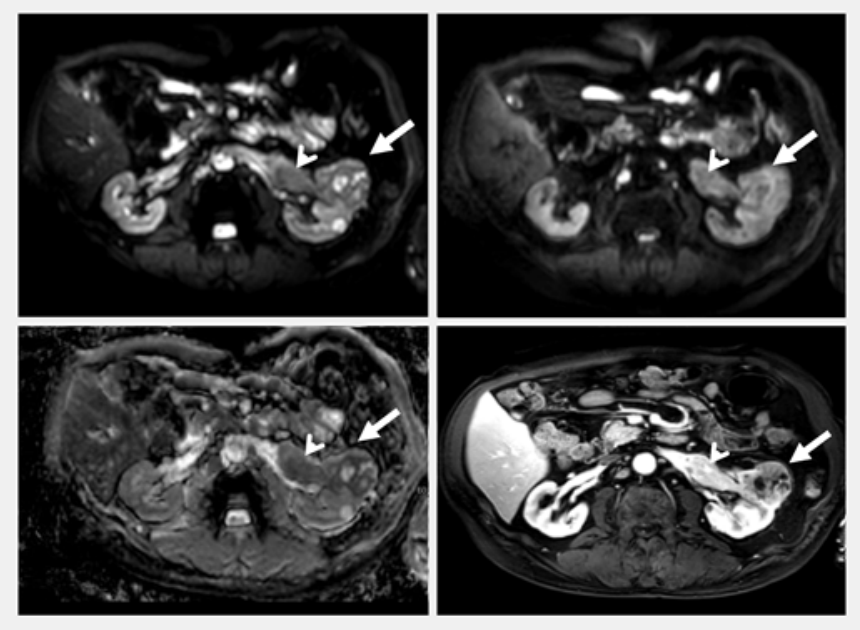

Abb. 14 80-jähriger Patient mit hellzelligem Nierenzellkarzinom (Pfeil) und Tumorthrombus (Pfeilspitze) in der linken Nierenvene. Im DWI-Bild (oberes linkes Bild, $\mathrm{b}=0 \mathrm{~s} / \mathrm{mm}^{2}$ ) ist eine Raumforderung der linken Niere mit zystischen Strukturen zu erkennen (Pfeil). Bei $\mathrm{b}=800 \mathrm{~s} / \mathrm{mm}^{2}$ (oberes rechtes Bild) sind die nicht diffusionsgestörten Zysten nicht mehr zu sehen. Aufgrund des Blutflusses verschwinden die großen Gefäße bis auf die linke Nierenvene (Pfeilspitze). Die ADCKarte (unteres linkes Bild) zeigt, dass Tumor und Tumorthrombus einen mittleren ADC-Wert (gemittelt anhand der b-Werte 0, 50, 250 und $800 \mathrm{~mm}^{2} / \mathrm{s}$ ) von $1,6 \times 10^{-3} \mathrm{~mm}^{2} / \mathrm{s}$ haben, der Bereich der ADC-Werte im partiell zystischen Karzinom beträgt dabei $0,5-2,7 \times 10^{-3} \mathrm{~mm}^{2} / \mathrm{s}$. Das untere rechte Bild zeigt die spätvenöse Phase der Kontrastmitteldynamik ebenfalls mit Karzinom und Tumorthrombus.

cherweise interessante Alternative könnte die rechnerische Simulation von DWI-Bildern mit höheren b-Werten sein. Allerdings ist diese Variante derzeit noch nicht auf allen MR-Systemen verfügbar ( $\triangleright$ Abb. 15).

In zahlreichen Studien ergab sich eine negative Korrelation zwischen dem ADC-Wert und der klinischen Aggressivität bzw. dem Gleason-Score, d.h. ein sehr niedriger ADC-Wert weist auf ein High-Risk-Karzinom 

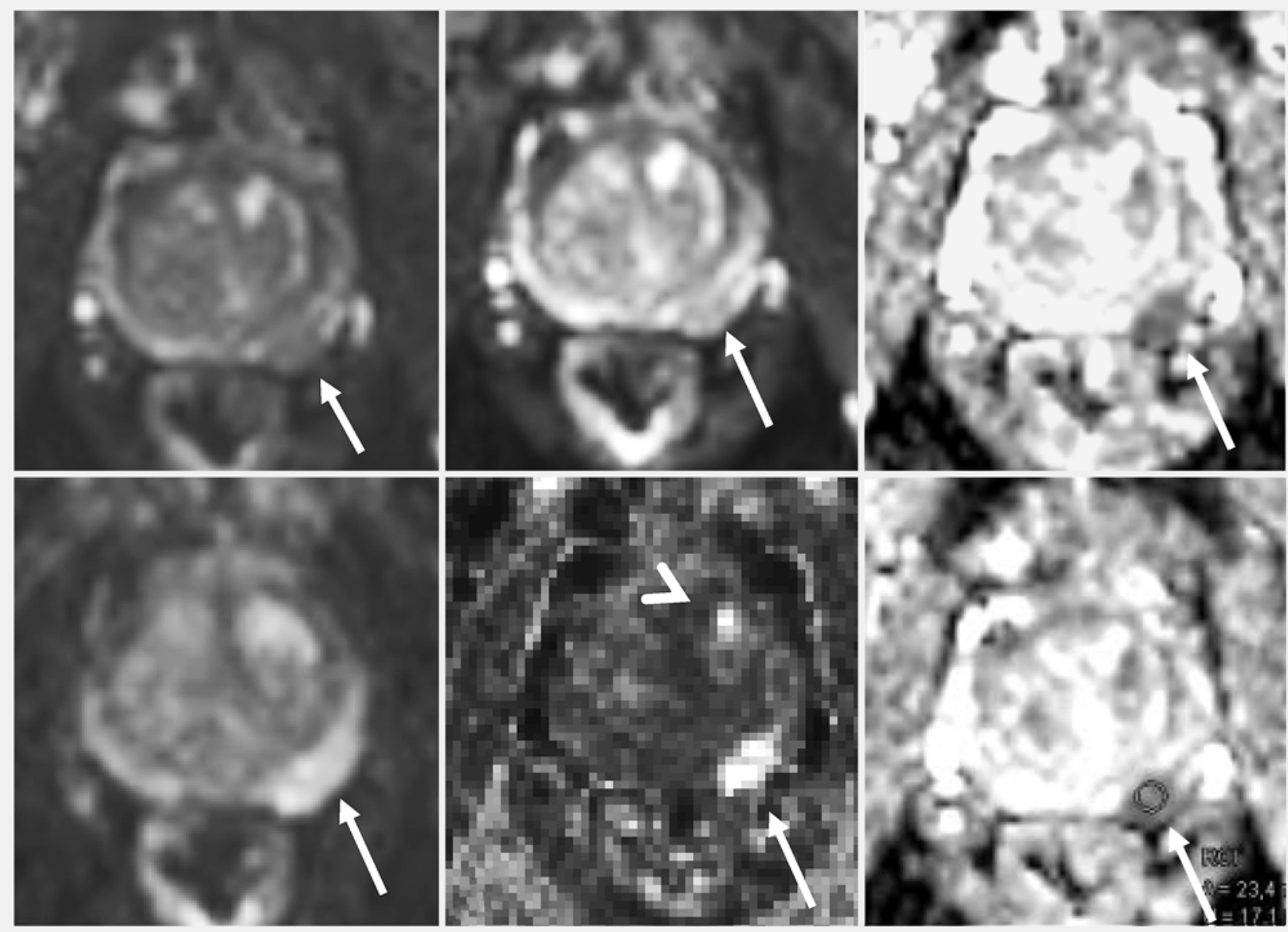

- Abb. 15 70-jähriger Patient mit histologisch gesichertem Prostatakarzinom in der linken Außenzone (Pfeil). Auf den DWI-Bildern (linke 4 Bilder) ist zu erkennen, dass das Karzinom mit zunehmendem b-Wert ( $b=0$ [linkes oberes Bild], 50 [linkes unteres Bild], $b=800 \mathrm{~s} / \mathrm{mm}^{2}$ [mittleres oberes Bild]) besser sichtbar wird. Bei einem rechnerischen b-Wert von $2000 \mathrm{~s} / \mathrm{mm}^{2}$ (mittleres unteres Bild) hebt sich das Karzinom deutlich besser vom nicht betroffenen Gewebe ab und eine weitere auffällige Region kommt besser zur Darstellung (Pfeilspitze). Die ADCKarte (rechte 2 Bilder), berechnet aus dem b-Werten 0 und 800 bzw. 50 und 800, zeigt das Karzinom mit einem mittleren ADC-Wert von $0,7 \times 10^{-3} \mathrm{~mm}^{2} / \mathrm{s}$.

bzw. einen hohen Gleason-Score hin. Obwohl aufgrund der histopathologischen Heterogenität von Prostatakarzinomen und der eingeschränkten Vergleichbarkeit der in verschiedenen Studien ermittelten ADC-Werte kein genereller Cut-off-Wert angegeben werden kann, sollte spätestens ab einem ADC-Wert von unter $800 \times$ $10^{-6} \mathrm{~mm}^{2} / \mathrm{s}$ das Prostatakarzinom erste und wichtigste Differenzialdiagnose sein.

Merke

Der ADC-Wert korreliert negativ mit der Aggressivität des Prostatakarzinoms und dem Gleason-Score. Der b-Wert sollte mindestens $800-1000 \mathrm{~s} / \mathrm{mm}^{2}$ betragen. Noch höhere (berechnete) b-Werte sind möglicherweise vorteilhaft.

\section{Lymphknoten}

\section{Allgemeines}

Der Nachweis von Lymphknotenmetastasen gehört aufgrund ihrer oft erheblichen therapeutischen und prognostischen Konsequenzen zu den wichtigsten Zielen des Tumor-Stagings. Die konventionelle Schnittbilddiagnostik liefert lediglich morphologische Kriterien wie Form, Größe und innere Architektur. Als funktionelle Technik kann die DWI prinzipiell zusätzliche Informationen liefern, allerdings ist die Diffusion in lymphatischem Gewebe generell eingeschränkt ( $\triangleright$ Abb. 16). Die DWI ist daher sehr gut geeignet, um Lymphknoten generell nachzuweisen. In Bezug auf die Unterscheidung zwischen Lymphknotenmetastasen und normalen Lymphknoten konzentrierten sich die bisher veröffentlichten Studien meist auf zervikale, mediastinale und pelvine Lymphknoten sowie auf das 
Staging des Lymphoms. Insgesamt ist die Datenlage sehr heterogen und teils widersprüchlich. Was die Anzahl und Auswahl der b-Werte angeht, besteht dementsprechend kein Konsens, sodass teils erhebliche Schwankungen der ADC-Grenzwerte zur Unterscheidung zwischen benignen und malignen Läsionen angegeben werden.

\section{Merke}

Die Datenlage zum Nachweis von Lymphknotenmetastasen und Lymphomen mit der DWI ist heterogen. Unstrittig ist, dass die DWI Lymphknoten jeglicher Dignität sehr gut nachweisen kann.

Mehrere Studien weisen statistisch signifikant unterschiedliche ADC-Werte zwischen benignen und malignen Lymphknoten nach, wobei in malignen Lymphknoten meist niedrigere ADC-Werte beobachtet wurden als in benignen, entzündlich veränderten Lymphknoten. Bei entzündlichen Erkrankungen wie z. B. der Tuberkulose, der Sarkoidose oder der Katzenkratzkrankheit zeigten entzündliche Lymphknoten jedoch auch niedrigere ADC-Werte als Metastasen, insbesondere dann, wenn sie relevante Mengen an fibrösem Gewebe aufwiesen. Zudem spielt es eine Rolle, ob nekrotische Zonen in die ADC-Messung eingeschlossen werden oder nicht. Nach einer aktuellen Metaanalyse liegen diagnostische Genauigkeit und negativer Vorhersagewert der DWI in Bezug auf Lymphknotenmetastasen jedoch bei etwa $90 \%$ [29].

\section{Merke}

In den meisten Studien weisen Lymphknotenmetastasen niedrigere ADC-Werte auf als benigne Lymphknoten - außer bei zentral nekrotischen Lymphknotenmetastasen. Allerdings haben auch benigne Lymphknoten manchmal niedrige ADC-Werte.

Gegenüber der FDG-PET bzw. PET-CT wies die DWI meist eine niedrigere Rate falsch positiver Lymphknoten und somit eine geringere Tendenz zum Over-Staging auf [8]. Daten zur DWI pelviner Lymphknoten sind uneinheitlich: Während manche Studien in Abhängigkeit des verwendeten Cut-Offs über eine gute Sensitivität und Spezifität von ca. $86 \%$ bzw. $85 \%$ bezüglich der Diagnose von Lymphknotenmetastasen beim Prostatakarzinom berichteten, zeigte eine Studie erheblich schlechtere Ergebnisse mit einer Sensitivität von ca. $19 \%$ bzw. einer Spezifität von etwa 46\% [30]. Demgegenüber wurde beim Staging des Rektumkarzinoms eine bessere Sensitivität und Spezifität durch die Kombination von DWI mit konventioneller MRT im Vergleich zur CT berichtet. Im Rahmen des Lymphom-Stagings dürfte die Diffusionsbildgebung aufgrund der fehlenden Strahlenbelastung besonders bei pädiatrischen Patienten oder im Rahmen längerer Nachsorge-Intervalle in Zukunft eine wichtige Rolle einnehmen.

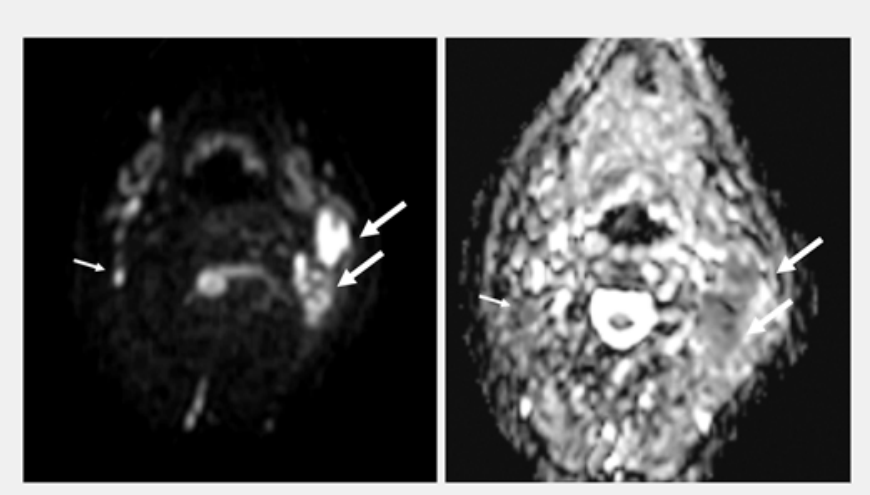

- Abb.16 56-jähriger Patient mit histologisch gesicherten Lymphknotenmetastasen eines Tonsillenkarzinoms. Das DWI-Bild (linkes Bild, b = $800 \mathrm{~s} / \mathrm{mm}^{2}$ ) zeigt die großen links zervikalen Metastasen (große Pfeile) sowie kleine unspezifische Lymphknoten auf der rechten Halsseite. Ausweislich der ADC-Karte (rechtes Bild) liegen die ADC-Werte sowohl in den Metastasen als auch in den übrigen unspezifischen Lymphknoten der Gegenseite bei etwa $0,5 \times 10^{-3} \mathrm{~mm}^{2} / \mathrm{s}$.

\section{KERNAUSSAGEN}

- Wassermoleküle im menschlichen Körper werden in ihrer Diffusion durch verschiedene Strukturen wie Zellmembranen und Makromoleküle gehindert. Grundlage der Diffusionsbildgebung sind die unterschiedlichen Eigenschaften verschiedener normaler und geschädigter Körperstrukturen in Hinsicht auf ihre Diffusionsrestriktion.

- Physiologische Bewegungen wie Atmung, Herzschlag, Pulsationen und Peristaltik sowie nicht physiologische Bewegungen haben einen großen Einfluss auf die Messgrößen in der Diffusionsbildgebung. Insbesondere die Einflüsse der Gewebeperfusion kann man sich dabei auch gezielt zunutze machen.

- Die Diffusionsbildgebung sollte grundsätzlich fettunterdrückt durchgeführt werden. Eine homogene Fettunterdrückung hat entscheidenden Einfluss auf die Bildqualität.

- Die Diagnostik des ischämischen Schlaganfalls, des Status epilepticus und die Abklärung entzündlicher Veränderungen sind eindeutige Indikationen der DWI im Bereich des ZNS. Sinnvoll ist die DWI zudem in der Lymphomdiagnostik einsetzbar.

- Im Bereich der Bildgebung von Thorax und Abdomen hat sich die DWI insbesondere in der Tumordiagnostik durchgesetzt. Ein breiter Konsens bezüglich der anzuwendenden Techniken - insbesondere der Wahl der b-Werte und der Art der ADCBerechnung - besteht jedoch noch nicht. 


\section{Interessenkonflikt}

Die Autoren geben an, dass kein Interessenkonflikt besteht.

\section{Über die Autoren}

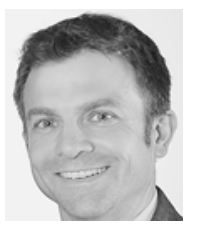

\section{Guido Matthias Kukuk}

Priv.-Doz. Dr. med. Studium der Humanmedizin in Bonn, Valencia und Zürich. Facharztausbildung zum Radiologen in Bonn. Seit 2014 Oberarzt der Radiologischen Klinik der Universitätsklinik Bonn. Klinische Schwerpunkte: MR-Tomografie, abdominelle und urogenitale Radiologie.

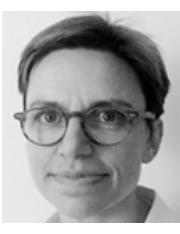

\section{Susanne Greschus}

Dr. med. Studium der Humanmedizin an der Friedrich-Wilhelms-Universität Bonn und der Westfälischen Wilhelms-Universität Münster. Facharztausbildung zur Ärztin für Radiologie und Neuroradiologie in den Universitätskliniken Gießen und Bonn. Seit 2012 Oberärztin in der Radiologischen Klinik der Universitätsklinik Bonn. Klinische Schwerpunkte: Neuroradiologie, Kopf-Hals-Diagnostik.

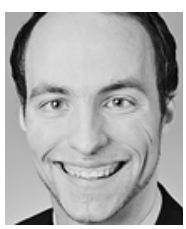

\section{Jan Goldstein}

Dr. med., M.A. 2003-2012 Studium der Humanmedizin und der Philosophie an der Universität Düsseldorf. 2013 Promotion an der Universität Ulm. 2012-2015 und seit 2016 Assistenzarzt am Institut für Diagnostische und Interventionelle Radiologie des Städtischen Klinikums Solingen, 2015-2016 Assistenzarzt an der Radiologischen Klinik des Universitätsklinikums Bonn.

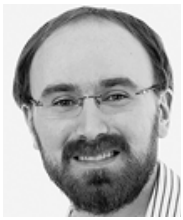

\section{Claus Christian Pieper}

Dr. med. 2005-2011 Studium der Humanmedizin an der Medizinischen Fakultät der Westfälischen Wilhelms-Universität Münster (WWU). Promotion an der WWU. Seit 2011 wissenschaftlicher Mitarbeiter in der Radiologischen Klinik der Rheinischen Friedrich-Wilhelms-Universität Bonn. Klinische und wissenschaftliche Schwerpunkte: Interventionelle Radiologie, onkologische Bildgebung, interventionelle Onkologie, Bildgebung und interventionelle Therapien des Lymphsystems.

\section{Korrespondenzadresse}

Priv.-Doz. Dr. med. Guido M. Kukuk

Radiologische Universitätsklinik Bonn

FE MRT

Sigmund-Freud-Straße 25

53127 Bonn

Tel.: 022828715874

E-Mail: Guido.Kukuk@ukb.uni-bonn.de
Danksagung

Wir danken Frau Dr. rer. nat. Petra Mürtz für die kritische Durchsicht des Manuskripts.

Literatur

11] Donnan GA, Baron J-C, Ma H et al. Penumbral selection of patients for trials of acute stroke therapy. Lancet Neurol 2009; 8: 261-269

[2] Olivot J-M, Mlynash M, Thijs VN et al. Relationships Between Cerebral Perfusion and Reversibility of Acute Diffusion Lesions in DEFUSE: Insights from RADAR. Stroke 2009; 40: $1692-1697$

[3] Al-Okaili RN, Krejza J, Wang S et al. Advanced MR Imaging Techniques in the Diagnosis of Intraaxial Brain Tumors in Adults. RadioGraphics 2006; 26: (Suppl. 01): S173-S189

[4] Schroeder PC, Post MJD, Oschatz E et al. Analysis of the utility of diffusion-weighted MRI and apparent diffusion coefficient values in distinguishing central nervous system toxoplasmosis from lymphoma. Neuroradiology 2006; 48 : $715-720$

[5] Vandecaveye V, De Keyzer F, Vander Poorten V et al. Head and Neck Squamous Cell Carcinoma: Value of Diffusionweighted MR Imaging for Nodal Staging 1. Radiology 2009; 251: $134-146$

[6] Regier M, Schwarz D, Henes FO et al. Diffusion-weighted MR-imaging for the detection of pulmonary nodules at 1.5 Tesla: Intraindividual comparison with multidetector computed tomography: Pulmonary Nodule detection at DW-MRI. J Med Imaging Radiat Oncol 2011; 55: 266-274

[7] Yang R-M, Li L, Wei X-H et al. Differentiation of Central Lung Cancer from Atelectasis: Comparison of Diffusion-Weighted MRI with PET/CT. PLoS ONE 2013; 8: e60279

[8] Razek AA. Diffusion magnetic resonance imaging of chest tumors. Cancer Imaging 2012; 12: 452

[9] Baysal T, Bulut T, Gökirmak M et al. Diffusion-weighted MR imaging of pleural fluid: differentiation of transudative vs exsudative pleural effusions. Eur Radiol 2004; 14: 890-896

[10] Parikh T, Drew SJ, Lee VS et al. Focal Liver Lesion Detection and Characterization with Diffusion-weighted MR Imaging: Comparison with Standard Breath-hold T2-weighted Imaging 1. Radiology 2008; 246: $812-822$

[11] Wu L-M, Hu J, Gu H-Y et al. Can diffusion-weighted magnetic resonance imaging (DW-MRI) alone be used as a reliable sequence for the preoperative detection and characterisation of hepatic metastases? A meta-analysis Eur J Cancer Oxf Engl 1990 2013; 49: 572 - 584

[12] Padhani AR, Koh D-M. Diffusion MR imaging for monitoring of treatment response. Magn Reson Imaging Clin N Am 2011; 19: $181-209$

[13] Karanjia RN, Crossey MME, Cox IJ et al. Hepatic steatosis and fibrosis: Non-invasive assessment. World J Gastroenterol 2016; 22: 9880

[14] Lewin M, Poujol-Robert A, Boëlle P-Y et al. Diffusionweighted magnetic resonance imaging for the assessment of fibrosis in chronic hepatitis C. Hepatology 2007; 46: $658-665$

[15] Park HJ, Kim SH, Jang KM et al. The role of diffusionweighted MR imaging for differentiating benign from malignant bile duct strictures. Eur Radiol 2014; 24: 947 -958 
[16] Robertis RD. Diffusion-weighted imaging of pancreatic cancer. World J Radiol 2015; 7: 319

[17] Wang Y, Chen ZE, Yaghmai V et al. Diffusion-weighted MR imaging in pancreatic endocrine tumors correlated with histopathologic characteristics. J Magn Reson Imaging 2011; 33: 1071 - 1079

[18] Wang Y, Miller FH, Chen ZE et al. Diffusion-weighted MR Imaging of Solid and Cystic Lesions of the Pancreas. RadioGraphics 2011; 31: E47-E64

[19] Seo N, Park SH, Kim K-J et al. MR enterography for the evaluation of small-bowel inflammation in Crohn disease by using diffusion-weighted imaging without intravenous contrast material: a prospective noninferiority study. Radiology 2015; 278: $762-772$

[20] Tielbeek JAW, Ziech MLW, Li Z et al. Evaluation of conventional, dynamic contrast enhanced and diffusion weighted MRI for quantitative Crohn's disease assessment with histopathology of surgical specimens. Eur Radiol 2014; 24: 619629

[21] Freiman M, Perez-Rossello JM, Callahan M] et al. Characterization of fast and slow diffusion from diffusion-weighted MRI of pediatric Crohn's disease. J Magn Reson Imaging 2013; 37: $156-163$

[22] Jha RC, Zanello PA, Ascher SM et al. Diffusion-weighted imaging (DWI) of adenomyosis and fibroids of the uterus. Abdom Imaging 2014; 39: 562 - 569

[23] Li HM, Liu J, Qiang JW et al. Diffusion-Weighted Imaging for Differentiating Uterine Leiomyosarcoma From Degenerated Leiomyoma. J Comput Assist Tomogr 2016: DOI: 10.1097| RCT.0000000000000565
[24] Ikink ME, Voogt M], van den Bosch MAAJ et al. Diffusionweighted magnetic resonance imaging using different $b$ value combinations for the evaluation of treatment results after volumetric MR-guided high-intensity focused ultrasound ablation of uterine fibroids. Eur Radiol 2014; 24 : $2118-2127$

[25] Nakayama T, Yoshimitsu K, Irie H et al. Diffusion-weighted echo-planar MR imaging and ADC mapping in the differential diagnosis of ovarian cystic masses: Usefulness of detecting keratinoid substances in mature cystic teratomas. J Magn Reson Imaging 2005; 22: 271-278

[26] Mürtz P, Flacke S, Träber F et al. Abdomen: Diffusionweighted MR Imaging with Pulse-triggered Single-Shot Sequences. Radiology 2002; 224: 258- 264

[27] Lassel EA, Rao R, Schwenke C et al. Diffusion-weighted imaging of focal renal lesions: a meta-analysis. Eur Radiol 2014; 24: $241-249$

[28] Zhang H, Gan Q, Wu Y et al. Diagnostic performance of diffusion-weighted magnetic resonance imaging in differentiating human renal lesions (benignity or malignancy): a meta-analysis. Abdom Radiol 2016; 41: 1997-2010

[29] Driessen JP, van Kempen PMW, van der Heijden G] et al. Diffusion-weighted imaging in head and neck squamous cell carcinomas: a systematic review. Head Neck 2015; 37: $440-448$

[30] Giannarini G, Petralia G, Thoeny HC. Potential and limitations of diffusion-weighted magnetic resonance imaging in kidney, prostate, and bladder cancer including pelvic lymph node staging: a critical analysis of the literature. Eur Urol 2012; 61: $326-340$

Bibliografie

DOI http://dx.doi.org/10.1055/s-0042-122605

Radiologie up2date 2017; 17: 83-103

(c) Georg Thieme Verlag KG Stuttgart · New York

ISSN 1616-0681

\section{ERRATUM}

Kukuk GM, Greschus S, Goldstein J, Pieper CC. Diffusionsbildgebung: Technik und klinische Anwendung. Radiologie up2date 2017; 17 (01): 83 - 103, DOI: 10.1055/s-0042122605

In die o.g. Arbeit hat sich leider ein durchgehender Fehler eingeschlichen.

Die Angabe der Maßeinheit beim ADC-Wert ist nicht korrekt und muss lauten: $\mathrm{mm}^{2} / \mathrm{s}$. (Die Maßeinheit beim b-Wert ist korrekt mit s/mm² im Artikel angegeben.)

Die Online-Version wurde im September 2017 korrigiert. 


\section{Punkte sammeln auf CME.thieme.de}

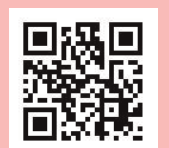

Diese Fortbildungseinheit ist 12 Monate online für die Teilnahme verfügbar.

Sollten Sie Fragen zur Online-Teilnahme haben, finden Sie unter http://cme.thieme.de|hilfe eine ausführliche Anleitung. Wir wünschen viel Erfolg beim Beantworten

der Fragen!

Unter eref.thieme.de/ZZWHP85 oder über den QR-Code kommen Sie direkt zum Artikel zur Eingabe der Antworten.

VNR 2760512017152372753

\section{Frage 1}

Welche der folgenden Aussagen zur Diffusionsbildgebung ist nicht richtig?

A Mit der DWI wird die Brown'sche Molekularbewegung auf dem MRT-Bild sichtbar gemacht.

B Bei der Standardsequenz nach Stejskal und Tanner werden starke Magnetfeldgradienten vor und nach einem Inversionsimpuls geschaltet. Bewegte Spins werden nicht vollständig rephasiert.

C Der b-Wert beschreibt u. a. die Gradientenstärke der diffusionsgewichteten Sequenz.

D Blutflussbewegungen haben im Allgemeinen keinen Einfluss auf das DWI-Signal.

E Um den Diffusionskoeffizienten D des Gewebes bestimmen zu können, müssen mindestens 2 Messungen mit unterschiedlichen b-Werten durchgeführt werden.

\section{Frage 2}

Die Diffusionsbildgebung hat mittlerweile einen großen Stellenwert in der MRT-Diagnostik. Welche der folgenden Aussagen ist richtig?

A Atmung und Herzbewegung sind unproblematisch für die Diffusionsbildgebung.

B Problematisch sind Organe mit kurzen T2-Relaxationszeiten.

C Der ADC-Wert ist ein quantitativer Parameter und hängt nicht vom b-Wert ab.

D Der ADC-Wert von Organen hängt nicht von ihrer Perfusion ab.

E Für die meisten Indikationen sind bereits Standards der DWI definiert.

\section{Frage 3}

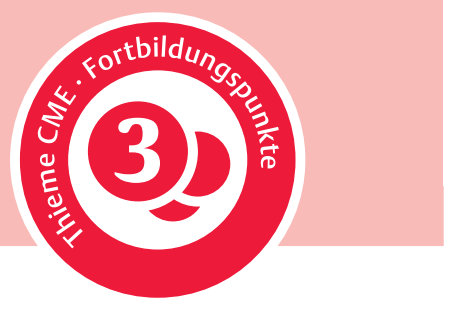

Welche der folgenden Aussagen ist nicht richtig?

A Die DWIBS-Technik eignet sich zum Staging und zur Verlaufskontrolle von Lymphom-Erkrankungen.

B Bei der DWIBS wird häufig kein ADC-Wert berechnet.

C Der Bildeindruck bei der DWIBS ist ähnlich zur PET.

D Die Analyse bei der DWIBS findet meist visuell-qualitativ an invertierten Bildern statt.

E Die DWIBS wird i.d. R. ohne Fettunterdrückung durchgeführt.

\section{Frage 4}

Welche der folgenden Aussagen zu Diffusionsstörungen beim Status epilepticus ist nicht richtig?

A Sie finden sich häufig in der Substantia nigra und im Kleinhirn.

B Die Diffusionsstörungen liegen oft einseitig kortikal und sparen das Marklager aus.

C Hippokampus und posteriorer Thalamus sind oft beteiligt.

D Die Diffusionsstörungen lassen sich keinem Gefäßterritorium zuordnen.

E Die Diffusionsstörungen sind meist reversibel.

\section{Frage 5}

Welche der folgenden Aussagen ist am ehesten richtig?

A Mit der DWI lassen sich intrakranielle Neoplasien und Hirnmetastasen gut voneinander abgrenzen.

B ZNS-Lymphome zeigen typischerweise höhere ADC-Werte als die zerebrale Toxoplasmose.

C Die DWI ist gut geeignet, um Epidermoidzysten von Arachnoidalzysten abzugrenzen.

D Der ADC-Wert ist nahezu proportional zur Tumorzelldichte, d. h. mit höherer Zelldichte steigt der ADC-Wert.

E In der Diagnostik einer Herpes-Enzephalitis hat die DWI derzeit keinen Stellenwert. 


\section{Punkte sammeln auf CME.thieme.de}

Fortsetzung ...

\section{Frage 6}

Welche der folgenden Aussagen ist nicht richtig?

A Nervenfaserbündel eignen sich aufgrund ihres hohen Organisationsgrades gut zur Darstellung im Rahmen der Diffusionstensorbildgebung.

B Lymphknotenmetastasen stellen sich i. d. R. hyperintens in der DWI dar.

C Aufgrund ihres hohen Flüssigkeitsgehalts weisen Abszesse i.d.R. keine Diffusionsstörung auf.

D Phlegmonöse Entzündungen sind i.d. R. nicht diffusionsgestört.

E Bei der Einschätzung der entzündlichen Aktivität von chronisch entzündlichen Darmerkrankungen erreicht die DWI die Genauigkeit der kontrastmittelverstärkten MRT.

\section{Frage 7}

Welche der folgenden Aussagen ist am ehesten richtig?

A Zum Nachweis pulmonaler Läsionen ist die DWI aufgrund der starken thorakalen Bewegungen nicht geeignet.

B Für das thorakale Lymphom-Staging ist die DWI geeignet und kann der PET-CT vergleichbare Ergebnisse liefern.

C Die DWI ist zur Unterscheidung zwischen malignen und benignen Rundherden gut geeignet.

D Die DWI liefert gute Ergebnisse für das lokale Staging des Ösophaguskarzinoms.

E Die DWI hat sich in der prognostisch wichtigen Unterscheidung der Pleuramesotheliom-Subtypen bereits als Standardmethode etabliert.

\section{Frage 8}

Welche der folgenden Aussagen ist nicht richtig?

A Zum Nachweis von Lebermetastasen ist die DWI sehr gut geeignet und anderen MRT-Sequenzen gleichwertig oder überlegen.

B Zur Charakterisierung von Leberläsionen sind DWI-Aufnahmen mit hohem b-Wert und die Analyse der ADC-Karte erforderlich.

C Leberhämangiome zeigen häufig einen T2-shine-throughEffekt.

D Zukünftig kann die DWI möglicherweise dabei helfen, zwischen benignen und malignen Gallengangsstrikturen zu unterscheiden.

E Ein Therapieansprechen von Lebermetastasen geht i.d.R. mit einem Abfall des ADC-Werts einher.

\section{Frage 9}

Welche der folgenden Aussagen ist nicht richtig?

A Die diffusionsgewichtete MRT ermöglicht die Beurteilung von entzündlichen Veränderungen beim Morbus Crohn.

B Uterusmyome weisen eine große morphologische Spannbreite auf, die sich in einem breiten Spektrum von ADCWerten widerspiegelt.

C Die diffusionsgewichtete MRT ist gut geeignet, um zwischen benignen und maligen Läsionen des Ovars zu differenzieren.

D Reife Teratome haben meist einen niedrigen ADC-Wert.

E Mit der DWI lässt sich ein Tumor der Cervix uteri besser vom nicht betroffenen oder ödematösen Gewebe abgrenzen.

\section{Frage 10}

Welche der folgenden Aussagen ist nicht richtig?

A Angiomyolipome haben als benigne Nierentumoren meist einen hohen ADC-Wert.

B Der ADC-Wert korreliert mit der Aggressivität des Prostatakarzinoms.

C Lymphknotenmetastasen lassen sich anhand ihres ADCWertes i.d.R. von entzündlich veränderten Lymphknoten unterscheiden.

D Die DWI ist die wichtigste Sequenz zur Diagnose der Prostatakarzinoms in der Außenzone.

E Eine Pulstriggerung führt an der Niere i. d. R. zu zuverlässigeren Messwerten mit geringerer Standardabweichung. 